\title{
Multistep Inhibition of $\alpha$-Synuclein Aggregation and Toxicity in Vitro and in Vivo by Trodusquemine
}

Michele Perni, ${ }^{\dagger,+,} \oplus$ Patrick Flagmeier, ${ }^{\dagger,+,} \oplus$ Ryan Limbocker, ${ }^{\dagger,+,+}$ Roberta Cascella, ${ }^{\S}$

Francesco A. Aprile, ${ }^{\dagger, \ddagger \odot ~ C e ́ l i n e ~ G a l v a g n i o n, ~}{ }^{\dagger, \| \bullet}$ Gabriella T. Heller, ${ }^{\dagger, \ddagger \odot}$ Georg Meisl, ${ }^{\dagger, \ddagger}$

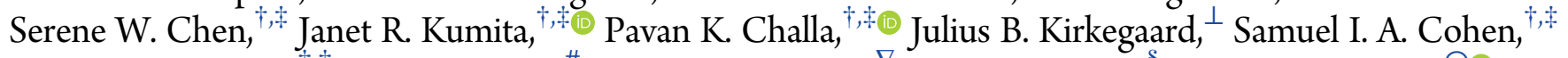
Benedetta Mannini, ${ }^{\dagger, \neq}$ Denise Barbut, ${ }^{\#}$ Ellen A. A. Nollen, ${ }^{\nabla}$ Cristina Cecchi, ${ }^{\S}$ Nunilo Cremades, ${ }^{\circ}$

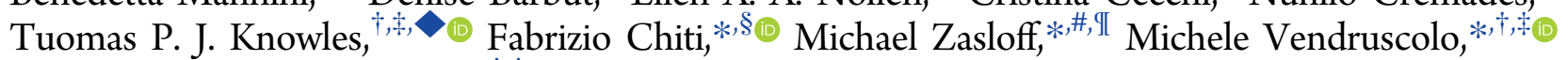
and Christopher M. Dobson* $*,+\neq \bullet$

${ }^{\dagger}$ Department of Chemistry, University of Cambridge, Cambridge CB2 1EW, United Kingdom

${ }^{\ddagger}$ Centre for Misfolding Diseases, Department of Chemistry, University of Cambridge, Cambridge CB2 1EW, United Kingdom

${ }^{\S}$ Department of Experimental and Clinical Biomedical Sciences, University of Florence, Florence 50134, Italy

${ }$ German Centre for Neurodegenerative Diseases, DZNE, Sigmund-Freud-Strasse. 27, 53127, Bonn, Germany

${ }^{\perp}$ Department of Applied Mathematics and Theoretical Physics, University of Cambridge, Cambridge CB3 0WA, United Kingdom

\#Enterin Inc., 3624 Market Street, Philadelphia, Pennsylvania 19104, United States

${ }^{\nabla}$ University Medical Centre Groningen, European Research Institute for the Biology of Aging, University of Groningen, Groningen 9713 AV, The Netherlands

OInstitute for Biocomputation and Physics of Complex Systems (BIFI)-Joint Unit BIFI-IQFR (CSIC), University of Zaragoza, 50018 Zaragoza, Spain

Department of Physics, Cavendish Laboratory, University of Cambridge, Cambridge CB3 0HE, United Kingdom

${ }^{\text {II } M e d S t a r-G e o r g e t o w n}$ Transplant Institute, Georgetown University School of Medicine, Washington, DC 20010, United States

\section{Supporting Information}

ABSTRACT: The aggregation of $\alpha$-synuclein, an intrinsically disordered protein that is highly abundant in neurons, is closely associated with the onset and progression of Parkinson's disease. We have shown previously that the aminosterol squalamine can inhibit the lipid induced initiation process in the aggregation of $\alpha$-synuclein, and we report here that the related compound trodusquemine is capable of inhibiting not only this process but also the fibril-dependent secondary pathways in the aggregation reaction. We further demonstrate that trodusquemine can effectively suppress the toxicity of $\alpha$-synuclein oligomers in neuronal cells, and that its administration, even after the initial

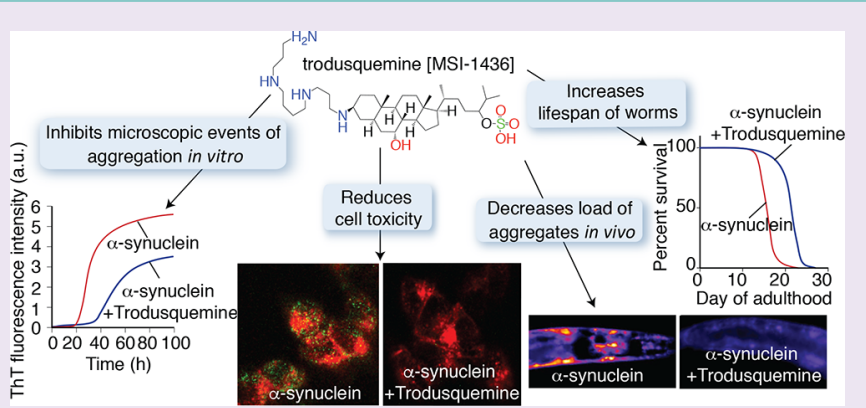
growth phase, leads to a dramatic reduction in the number of $\alpha$-synuclein inclusions in a Caenorhabditis elegans model of Parkinson's disease, eliminates the related muscle paralysis, and increases lifespan. On the basis of these findings, we show that trodusquemine is able to inhibit multiple events in the aggregation process of $\alpha$-synuclein and hence to provide important information about the link between such events and neurodegeneration, as it is initiated and progresses. Particularly in the light of the previously reported ability of trodusquemine to cross the blood-brain barrier and to promote tissue regeneration, the present results suggest that this compound has the potential to be an important therapeutic candidate for Parkinson's disease and related disorders.

$\mathrm{T}^{\mathrm{k}}$ he aggregation of $\alpha$-synuclein, a 140-residue protein highly expressed in neuronal synapses, ${ }^{1-5}$ is a hallmark of the pathogenesis of a variety of neurodegenerative disorders collectively known as $\alpha$-synucleinopathies, including Parkinson's disease (PD), PD with dementia, dementia with Lewy bodies, and multiplesystem atrophy. ${ }^{5-12}$ The mechanism of aggregation of $\alpha$-synuclein is highly complex and is modulated by a variety of environmental factors, such as $\mathrm{pH}$, temperature, ionic strength, and the presence of cosolvents, and by its interactions with a range of cellular components, including lipid membranes. ${ }^{13-20}$ In addition, the aggregation process is highly heterogeneous and leads to the formation of multiple types of fibrillar and prefibrillar species, the degree of polymorphism of which also depends on the experimental conditions. ${ }^{21-26}$

Received: May 18, 2018

Accepted: June 4, 2018

Published: June 28, 2018 
Although it is challenging to study the mechanistic events associated with $\alpha$-synuclein aggregation, a detailed understanding of this process is of considerable importance for the rational development and evaluation of potential therapeutics directed at reducing or eliminating the underlying sources of toxicity that lead to PD. ${ }^{10,27-29}$ The aggregation of $\alpha$-synuclein has been shown to be enhanced dramatically by its binding to lipid membranes; ${ }^{14,30}$ disrupting such interactions with small molecules therefore, has the potential to provide new information about the molecular processes involved in pathogenicity and could also represent the basis for an effective therapeutic strategy. In this context, we have recently found that the aminosterol squalamine ${ }^{31-33}$ interferes with the binding of $\alpha$-synuclein to membranes, reduces the initiation of its aggregation in vitro, and decreases the toxicity associated with such aggregation in human neuroblastoma cells and in a C. elegans model of PD. ${ }^{29}$

The existence of additional natural aminosterol compounds related to squalamine ${ }^{33}$ prompted us to explore their effects on the formation and properties of $\alpha$-synuclein aggregates in vitro and in vivo. One such compound, trodusquemine (also known as MSI-1436, Figure S1a), ${ }^{32-34}$ belongs to a class of cationic amphipathic aminosterols that have been widely studied in both animal models and in clinical trials in relation to the treatment of cancer, ${ }^{35}$ anxiety, ${ }^{36}$ and obesity. ${ }^{37,38}$ Trodusquemine was initially isolated from dogfish shark liver as a minor aminosterol along with six other related compounds, including squalamine. ${ }^{33}$ Moreover, it has since been shown to be able to cross the blood-brain barrier, a property of considerable importance for any therapeutic molecule for the treatment of neuropathic disorders. ${ }^{31}$ In addition, trodusquemine has been shown to be able to stimulate regeneration in a range of vertebrate tissues and organs following injury with no apparent effect on uninjured tissues. ${ }^{39}$ Trodusquemine shares the same parent structure as squalamine, but with a spermine moiety replacing the spermidine on the side chain, resulting in an increased positive charge (Figure S1a).

Given the structural similarity between trodusquemine and squalamine, and previous reports suggesting that the more positively charged trodusquemine is likely to enhance its ability to reduce negative electrostatic surface charges on intracellular membranes, ${ }^{32,40}$ we set out to characterize the effects of trodusquemine on the aggregation kinetics of $\alpha$-synuclein in vitro using experimental conditions previously used to study individual microscopic steps in the aggregation of $\alpha$-synuclein in the absence of any aminosterol. ${ }^{15,41}$

Our first studies of the effects of trodusquemine presented here were aimed to probe its effects on the initiation of $\alpha$-synuclein aggregation in the presence of lipid vesicles, ${ }^{14,15,30,41}$ on fibril elongation, ${ }^{13,15,41}$ and on secondary nucleation. ${ }^{13,15,41,42}$ We then explored the effects of trodusquemine on inhibiting the cytotoxicity of preformed oligomers of $\alpha$-synuclein toward neuronal cells. ${ }^{43}$ We also evaluated the effects of trodusquemine using a well-established transgenic $C$. elegans model of PD, in which $\alpha$-synuclein forms inclusions over time in the large muscle cells leading to age-dependent paralysis. ${ }^{29}$

\section{RESULTS AND DISCUSSION}

Trodusquemine Inhibits Both the Lipid-Induced Initiation and the Fibril-Induced Amplification Steps of $\alpha$-Synuclein Aggregation. In order to characterize systematically the influence of trodusquemine on the microscopic events involved in the aggregation of $\alpha$-synuclein, we employed a previously described three-pronged chemical kinetics strategy. ${ }^{13,15,41,42}$ This approach involves the study of $\alpha$-synuclein aggregation under a series of specifically designed conditions that make it possible to characterize separately the processes of heterogeneous primary nucleation, ${ }^{14}$ fibril elongation, ${ }^{13,15,41,42}$ and fibril amplification, ${ }^{13,15,41,42}$ the latter including secondary processes such as fragmentation and surface-catalyzed secondary nucleation that result in the proliferation of aggregated forms of $\alpha$-synuclein.

First, we tested the influence of trodusquemine on the lipidinduced initiation of $\alpha$-synuclein aggregation. ${ }^{14,15}$ Using 1,2Dimyristoyl-sn-glycero-3-phospho-L-serine (DMPS) vesicles at $30{ }^{\circ} \mathrm{C}$, ${ }^{14}$ we observed that increasing concentrations of trodusquemine resulted in an increase in the diameter of the vesicles to above $100 \mathrm{~nm}$ for trodusquemine-to-lipid ratios above 0.2 , as monitored by dynamic light scattering (Figure S2a). We have shown previously that variations in the size of the vesicles below $100 \mathrm{~nm}$ does not affect the kinetics of aggregation of $\alpha$-synuclein, ${ }^{14}$ and so in the present study we carried out all kinetic and lipidbinding experiments at trodusquemine-to-lipid ratios below 0.2 . $\alpha$-Synuclein was therefore incubated at a variety of concentrations, ranging from 20 to $100 \mu \mathrm{M}$, in the presence of $100 \mu \mathrm{M}$ DMPS under quiescent conditions at $30{ }^{\circ} \mathrm{C}$, and in the presence of concentrations of trodusquemine ranging from 0 to $10 \mu \mathrm{M}$. The aggregation reaction was monitored in real time using ThT fluorescence (Figures S3 and S4), and we observed a dose-dependent inhibition of the lipid-induced aggregation of $\alpha$-synuclein by trodusquemine, which was very similar to that observed in the presence of squalamine. ${ }^{29}$

In light of these results, we investigated the influence of trodusquemine on the lipid-binding properties of $\alpha$-synuclein using far-UV circular dichroism (CD) spectroscopy. We incubated $5 \mu \mathrm{M} \alpha$-synuclein in the presence of $250 \mu \mathrm{M}$ DMPS and increasing concentrations of trodusquemine $(0-50 \mu \mathrm{M}$; Figure S2b,c). At the protein-to-lipid ratio used in these experiments, effectively all the protein molecules in the absence of trodusquemine are bound to the surface of DMPS vesicles in an $\alpha$-helical conformation, as predicted from the binding constants previously determined for the $\alpha$-synuclein-DMPS system ${ }^{14}$ (Figure S2b). In the presence of increasing concentrations of trodusquemine, the CD signal of $\alpha$-synuclein measured at $222 \mathrm{~nm}$ increases from a value characteristic of an $\alpha$-helix to that of a random coil (Figure S2c), indicating that trodusquemine, like squalamine, can displace $\alpha$-synuclein from the surfaces of vesicles.

We then analyzed these data using the competitive binding model that was used to describe the displacement of $\alpha$-synuclein from vesicles by $\beta$-synuclein ${ }^{13,15,41}$ and by squalamine, ${ }^{29}$ in which $\alpha$-synuclein and $\beta$-synuclein or squalamine compete for binding sites at the surface of the DMPS vesicles. This analysis allowed us to determine both the dissociation constant $\left(K_{\mathrm{D}, \mathrm{T}}\right)$ and stoichiometry $\left(L_{\mathrm{T}}\right)$ associated with the trodusquemine-DMPS system, $K_{\mathrm{D}, \mathrm{T}}=1.62 \times 10^{-8} \mathrm{M}$ and $L_{\mathrm{T}}=5.1$ (Figure S2c). In comparable experiments conducted with the squalamine-DMPS system, we found $K_{\mathrm{D}, \mathrm{S}}=6.7 \times 10^{-8} \mathrm{M}$ and $L_{\mathrm{S}}=7.3$, respectively; ${ }^{29}$ the higher affinity for the anionic phospholipids by trodusquemine compared to squalamine is expected on the basis of the higher net positive charge of the former zwitterion. To characterize this inhibition in more detail, we analyzed the early time points of the aggregation reaction (see Materials and Methods for details) by globally fitting a single-step nucleation model to the kinetic traces as previously described for squalamine ${ }^{14}$ (Figures 1a,b, S4 and S5). This analysis is described in the Supporting Information (SI) and indicates that trodusquemine inhibits $\alpha$-synuclein lipid induced aggregation via a somewhat more complex mechanism than that reported for squalamine. In particular, our analysis suggests that the mechanism involves not only the displacement of 
a

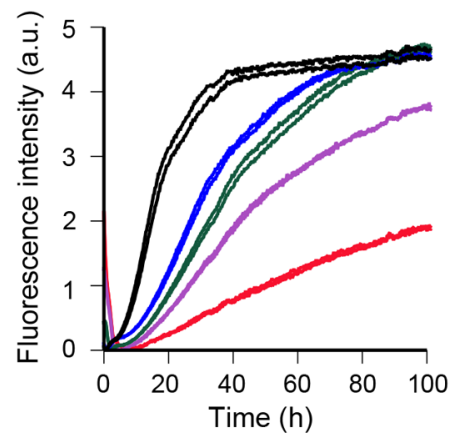

b

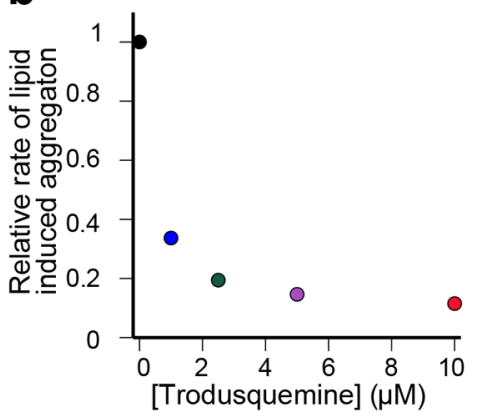

c
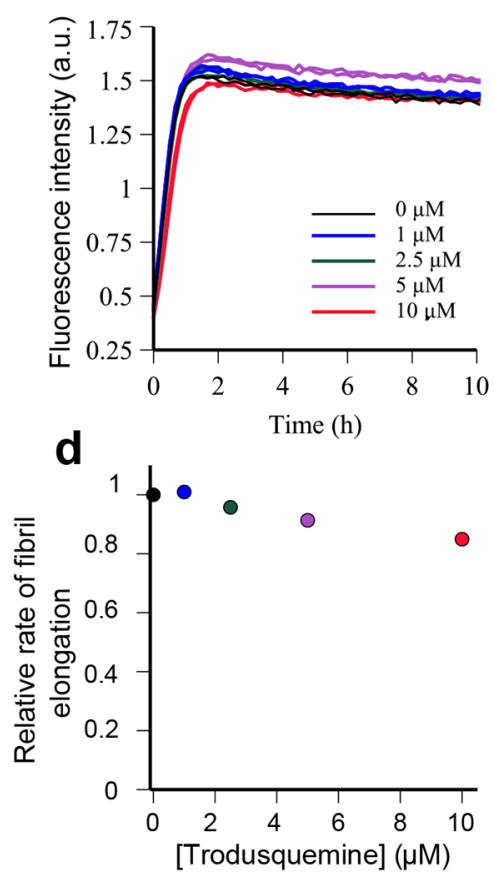

e

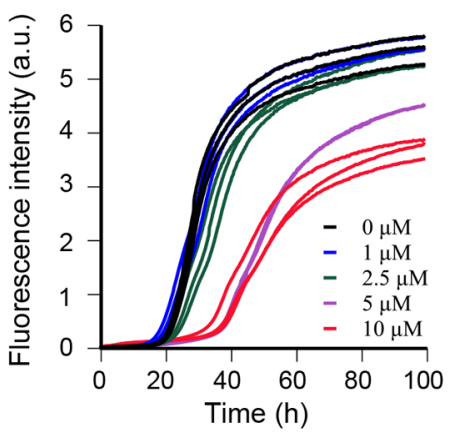

f

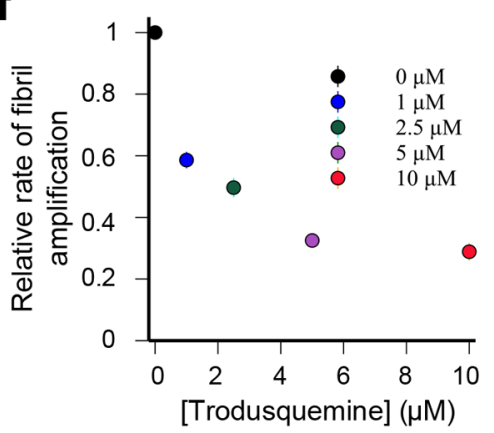

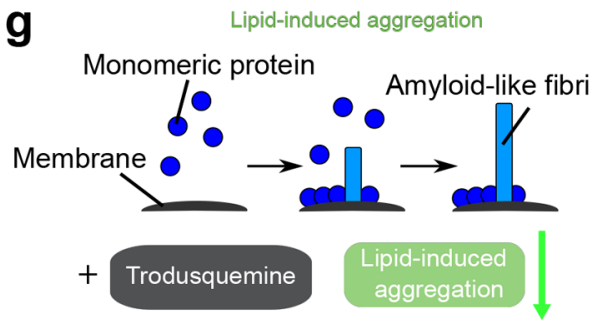
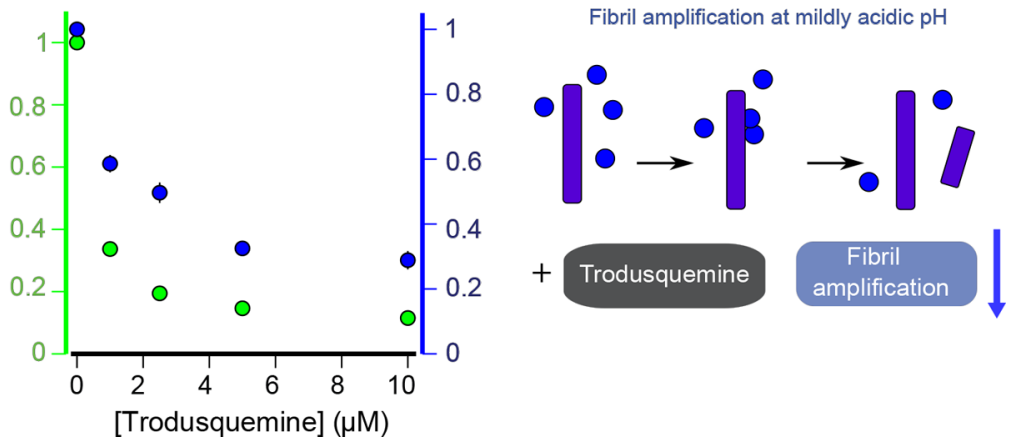

[Trodusquemine] $(\mu \mathrm{M})$

Figure 1. Inhibition of both the lipid-induced initiation and the fibril-induced amplification steps in $\alpha$-synuclein aggregation in vitro by trodusquemine. (a) Change in ThT fluorescence intensity when $100 \mu \mathrm{M}$ monomeric $\alpha$-synuclein was incubated in the presence of $100 \mu \mathrm{M}$ DMPS vesicles and $50 \mu \mathrm{M}$ ThT in $20 \mathrm{mM}$ phosphate buffer ( $\mathrm{pH} 6.5)$ under quiescent conditions at $30^{\circ} \mathrm{C}$. Trodusquemine was added to the solutions at increasing concentrations (black, $0 \mu \mathrm{M}$; dark blue, $1 \mu \mathrm{M}$; green, $2.5 \mu \mathrm{M}$; purple, $5 \mu \mathrm{M}$; red, $10 \mu \mathrm{M}$ ); two independent traces are shown for each concentration. (b) Relative rates of lipid-induced aggregation. The data were analyzed by globally fitting the early times of the kinetic traces using a single-step nucleation model ${ }^{15,41}$ (see Materials and Methods for details). (c) Change in ThT fluorescence intensity when $100 \mu \mathrm{M}$ monomeric $\alpha$-synuclein was incubated in the presence of $5 \mu \mathrm{M}$ preformed fibrils (monomer equivalents) and $50 \mu \mathrm{M}$ ThT in $20 \mathrm{mM}$ phosphate buffer (pH 6.5) under quiescent conditions at $37^{\circ} \mathrm{C}$. Increasing concentrations of trodusquemine were added to the solution (colors as in panel a), and two independent traces are shown for each concentration. (d) Effects of trodusquemine on the relative rates of fibril elongation. The rates of elongation were extracted through linear fits to the early time points of the kinetic traces shown in c. ${ }^{13,15,41}$ (e) Change in ThT fluorescence when $100 \mu \mathrm{M}$ monomeric $\alpha$-synuclein was incubated in the presence of $50 \mathrm{nM}$ preformed fibrils and $50 \mu \mathrm{M}$ ThT in $20 \mathrm{mM}$ phosphate buffer $(\mathrm{pH} 4.8)$ at $37^{\circ} \mathrm{C}$ under quiescent conditions. Trodusquemine was added at different concentrations (colors as in panel a; two independent traces are shown for each concentration. (f) Effects of trodusquemine on the relative rates of fibril amplification. The rates were analyzed by determining the change in fibril number concentration at the half time of the aggregation reaction. ${ }^{15,41}$ (g) Schematic representation of the effects of trodusquemine on lipid-induced aggregation and fibril amplification.

monomeric $\alpha$-synuclein from the membrane, as observed for squalamine, but also the interaction with intermediate species on the aggregation pathway; indeed, such interactions could also contribute to the ability of trodusquemine to suppress the interaction of $\alpha$-synuclein oligomers with cell membranes as described below.

We next explored the influence of trodusquemine on fibril elongation and secondary nucleation using experiments carried out in the presence of preformed fibrils at neutral and acidic $\mathrm{pH} .{ }^{13,15,41}$ We studied fibril elongation by performing experiments with monomeric $\alpha$-synuclein $(20-100 \mu \mathrm{M})$ in the presence of preformed fibrils of the protein at a concentration of $5 \mu \mathrm{M}$ (monomer equivalents) under quiescent conditions at $\mathrm{pH} 6.5$ and $37{ }^{\circ} \mathrm{C}$ (Figures 1c and S6); under these conditions, the kinetics of $\alpha$-synuclein aggregation have been found to be dominated by the rate of fibril elongation. ${ }^{13,15}$ The kinetic profiles of the aggregation reaction were then acquired in the presence of various concentrations of trodusquemine $[0-10 \mu \mathrm{M}]$. In all cases, the ThT fluorescence was found to decrease slowly during the apparent plateau reached at the end of the reaction. This behavior is often observed in such measurements, primarily because fibrils formed during the reaction tend to assemble into large assemblies with reduced exposed surface area available for ThT interaction. ${ }^{13,15,41}$ For each trodusquemine concentration, 
we extracted the elongation rate through linear fits of the early time points of the kinetic traces ${ }^{13,15}$ and found that trodusquemine does not detectably influence fibril elongation (Figures $1 \mathrm{~d}$ and S7). We then explored the influence of trodusquemine on fibrilcatalyzed secondary nucleation by incubating monomeric $\alpha$-synuclein $(60-100 \mu \mathrm{M})$ at $37^{\circ} \mathrm{C}$ in the presence of preformed fibrils at a concentration of $50 \mathrm{nM}$ (monomer equivalents) with increasing concentrations of trodusquemine $(0-10 \mu \mathrm{M})$ under quiescent conditions at $\mathrm{pH} 4.8^{13,15,41}$ (Figures 1e,f, S8, S9). We then analyzed the change in fibril number concentration as previously described ${ }^{15}$ and found that the rate of secondary nucleation decreased significantly with increasing concentrations of trodusquemine.

The observation that trodusquemine can inhibit secondary nucleation processes is likely to be associated with its ability to bind to the surfaces of amyloid fibrils, as such a situation has been found for molecular chaperones that show similar abilities. ${ }^{44} \mathrm{We}$ therefore probed the binding of trodusquemine to $\alpha$-synuclein fibrils by incubating preformed fibrils at a concentration of $10 \mu \mathrm{M}$ (monomer equivalents) overnight with equimolar concentrations of trodusquemine, followed by an ultracentrifugation step. ${ }^{29} \mathrm{We}$ determined the concentration of trodusquemine in the supernatant before and after incubation with fibrils using mass spectrometry (Figure S10) and found that approximately $70 \%$ of the trodusquemine molecules were associated with the fibrils, consistent with its ability to bind to their surfaces and inhibit the secondary nucleation of $\alpha$-synuclein. In summary, therefore, we observed that trodusquemine inhibits both the lipid-induced initiation and the fibril-induced amplification (secondary nucleation) step, in the process of $\alpha$-synuclein aggregation (Figure $1 \mathrm{~g}$ ). This behavior can be attributed, at least in large part, to its ability to displace the protein from the surface of both lipid vesicles and fibrils.

In order to exclude the probability that any contribution to the observed effects was a result of the quenching of ThT by trodusquemine, we incubated preformed $\alpha$-synuclein fibrils with $\mathrm{ThT}$ in the presence or absence of trodusquemine and could see that the compound did not affect the ThT signal to any detectable extent (Figure S11). In addition, because it has been found in some cases that a small molecule can inhibit the aggregation by sequestering proteins in a nonspecific manner as micelles or larger aggregates, ${ }^{45}$ we investigated the behavior of trodusquemine itself under the conditions used here $(20 \mathrm{mM}$ phosphate buffer, $\mathrm{pH} 6.5,30{ }^{\circ} \mathrm{C}$ ), using $1 \mathrm{D}^{1} \mathrm{H}$ and $2 \mathrm{D}{ }^{1} \mathrm{H}$ diffusion ordered spectroscopy (DOSY) experiments (Figures S12 and S13). The comparison of the trodusquemine intensities with those of an internal standard with the same concentration indicated that trodusquemine is largely $(>95 \%)$ soluble under these conditions (Figure S12). Moreover, a diffusion coefficient of $2.42 \times 10^{-10} \mathrm{~m}^{2} \mathrm{~s}^{-1}$, which is typical of a small molecule of this size in an aqueous solution, was determined for trodusquemine under these conditions, ruling out its self-assembly in solution (Figure S13). This result is supported by the absence of significant effects in the elongation step described above (Figure 1), which would be decreased if the concentration of free monomeric trodusquemine were reduced.

Trodusquemine Suppresses the Toxicity of $\alpha$-Synuclein Oligomers in Human Neuroblastoma Cells by Inhibiting Their Binding to the Cell Membrane. In an additional series of experiments, we explored the effect of trodusquemine on the cytotoxicity associated with the aggregation of $\alpha$-synuclein. ${ }^{43,46,47}$ We prepared samples of toxic type B* oligomers based on recently developed protocols ${ }^{46,47}$ and added them to the cell culture medium of human SH-SY5Y neuroblastoma cells at a concentration of $0.3 \mu \mathrm{M}$ (monomer equivalents of $\alpha$-synuclein). The MTT assay, which provides a measure of cellular viability (see Methods for details), confirms previous results that these oligomers are toxic to cells (Figure 2a). We then treated the cells with these oligomers $(0.3 \mu \mathrm{M})$ in the presence of increasing concentrations of trodusquemine $(0.03,0.1$, and $0.3 \mu \mathrm{M})$ and observed that the toxicity was markedly reduced, particularly at the highest trodusquemine concentration $(0.3 \mu \mathrm{M})$ where essentially complete protection was observed (Figure $2 \mathrm{a}$ ). In addition, these $\alpha$-synuclein oligomers $(0.3 \mu \mathrm{M})$ in the absence of trodusquemine were shown to induce an increase in the levels of reactive oxygen species (ROS) in this cell model, indicating their ability to inflict cellular damage (Figure $2 b$ ). Repeating the experiments with increasing concentrations of trodusquemine $(0.03,0.3$, and $3.0 \mu \mathrm{M})$, however, resulted in a marked decrease in the degree of ROS-derived fluorescence, showing a welldefined dose dependence and virtually complete inhibition of intracellular ROS production at a protein-to-trodusquemine ratio of 1:10 (Figure $2 \mathrm{~b}$ ).

We next investigated the mechanism by which trodusquemine inhibits $\alpha$-synuclein oligomer toxicity by probing the interactions between the oligomers $(0.3 \mu \mathrm{M})$ and human SH-SY5Y cells at increasing concentrations of trodusquemine $(0.03,0.3$, and $3.0 \mu \mathrm{M})$ using anti- $\alpha$-synuclein antibodies in conjunction with confocal microscopy. The images were scanned at apical planes to detect oligomers (green) interacting with cellular surfaces (red) by confocal microscopy (Figure 2c). Following the addition of the $\alpha$-synuclein oligomers to the cell culture medium, a large number of these species were observed to be associated with the plasma membranes of the cells, but their number was significantly and progressively decreased as the trodusquemine concentration was increased, showing a well-defined dose dependence (Figure 2c). We have shown previously that the toxicity caused by protein oligomers, which are membrane disruptive, $43,47,48$ correlates with the affinity of membrane binding. ${ }^{49}$ This protective effect can therefore be attributed to the reduced ability of $\alpha$-synuclein oligomers to interact with the cell membranes in the presence of trodusquemine. Such protection is likely to result from the ability of trodusquemine to bind to the cellular membranes and displace the oligomers from them, and potentially through its interactions with the oligomeric species themselves as suggested by the in vitro experiments on lipid binding discussed above.

Trodusquemine Inhibits Formation of the $\alpha$-Synuclein Inclusion in a C. elegans Model of PD and Increases Both Fitness and Longevity of the PD Worms. To examine whether or not trodusquemine shows the effects observed in cell cultures in a living organism, we used a well-established C. elegans model in which $\alpha$-synuclein is overexpressed in the large muscle cells ("PD worms") that shows age-dependent inclusion formation and related toxicity, which can be measured by a decrease in the number of body bends per minute (BPM), an increase in paralysis rate, and a decrease in the speed of movement. ${ }^{29}$ As described previously for squalamine, we first carried out experiments aimed at optimizing the treatment profile of the worms. ${ }^{29}$ We evaluated different treatment schedules by administering trodusquemine as a single, continuous dose either at an early stage of the life of the animals (L4 larval stage) or late in adulthood (D5, adulthood stage; Figure 3a).

We then investigated PD worms expressing $\alpha$-synuclein fused to the yellow fluorescent protein (YFP), and also control worms expressing only YFP, in conjunction with fluorescence microscopy 
a

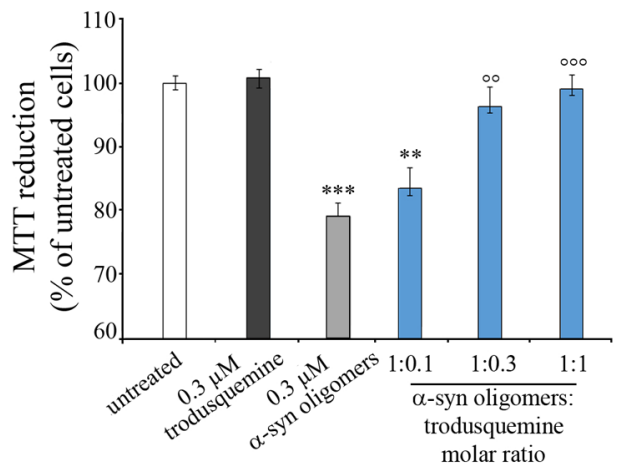

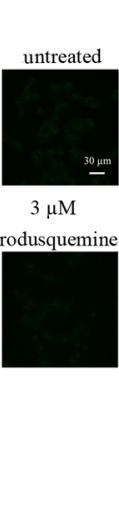

$\alpha$-syn oligomers:trodusquemine
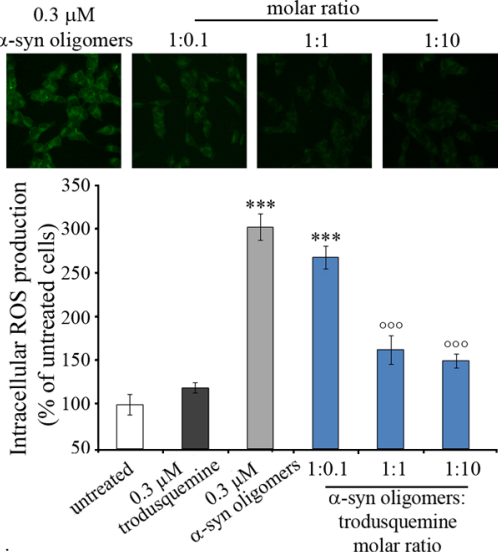

c

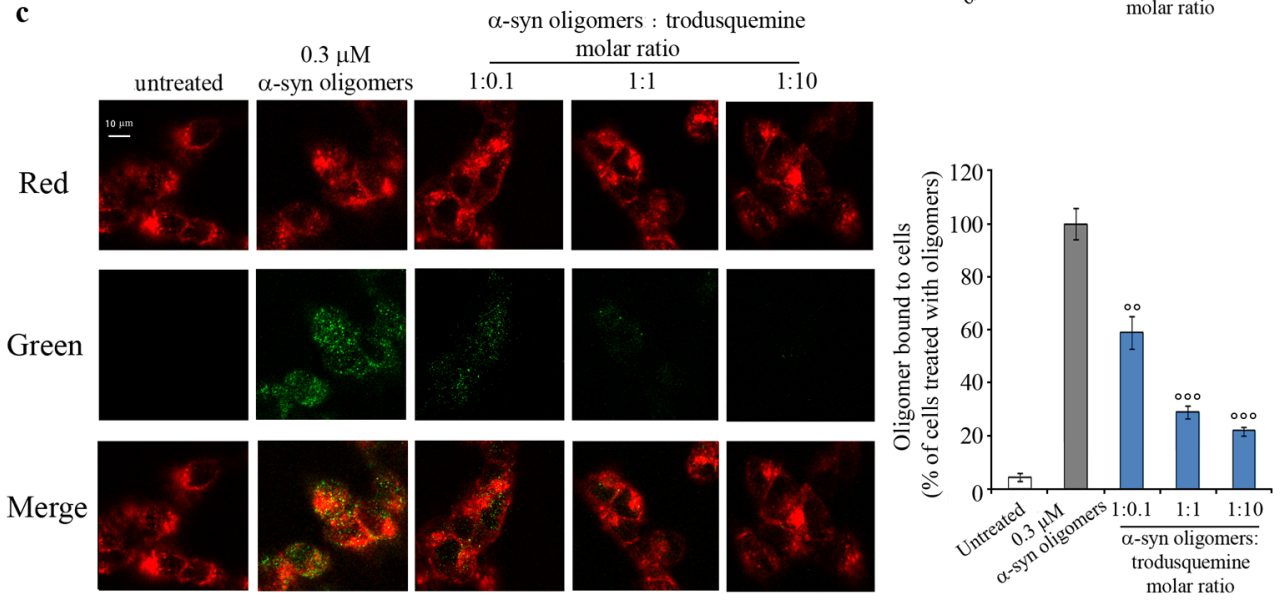

Figure 2. Trodusquemine suppression of the toxicity of $\alpha$-synuclein oligomers in human neuroblastoma SH-SY5Y cells by inhibition of their binding to cell membranes. (a) Type B* $\alpha$-synuclein oligomers were resuspended in the cell culture medium at a concentration of $0.3 \mu \mathrm{M}$ (monomer equivalents), incubated with or without different concentrations $(0.03,0.1$, and $0.3 \mu \mathrm{M})$ of trodusquemine for $1 \mathrm{~h}$ at $37^{\circ} \mathrm{C}$ under shaking conditions and then added to the cell culture medium of SH-SYSY cells for $24 \mathrm{~h}$ to test the ability of the cells to reduce MTT. Experimental errors are SEM $* * P \leq 0.01$ and $* * * P \leq$ 0.001 , respectively, relative to untreated cells. ${ }^{\circ} \mathrm{P} \leq 0.01$ and ${ }^{\circ 0}{ }^{\circ} \mathrm{P} \leq 0.001$, respectively, relative to cells treated with $\alpha$-synuclein oligomers. (b) Top panel: Representative confocal scanning microscope images of SH-SY5Y cells showing the levels of intracellular ROS following a 15 min incubation with $0.3 \mu \mathrm{M} \alpha$-synuclein type $\mathrm{B}^{*}$ oligomers (monomer equivalents) in the absence or presence of $0.03,0.3$, and $3.0 \mu \mathrm{M}$ trodusquemine. The green fluorescence arises from the CM- $\mathrm{H}_{2}$ DCFDA probe reacting with ROS. Lower panel: quantification. ${ }^{*}$ and ${ }^{\circ}$ symbols as in panel A. (c) Representative confocal scanning microscopy images of the apical sections of SH-SY5Y cells treated for 15 min with $\alpha$-synuclein of type $\mathrm{B}^{*}$ oligomers $(0.3 \mu \mathrm{M}$ monomer equivalents) and different concentrations $(0.03,0.3$, or $3.0 \mu \mathrm{M})$ of trodusquemine (left panels). Red and green fluorescence indicate the cell membranes and the $\alpha$-synuclein oligomers, respectively. Right: quantification of oligomer binding to the cells as a fraction of the cells treated with oligomers (right panel). ${ }^{\circ}$ and ${ }^{\circ \circ}$ symbols as in panel A.

in order to probe the number and distribution of $\alpha$-synuclein inclusions. ${ }^{50,51}$ The expression of YFP in the large muscle cells of the control animals was uniform and did not lead to the formation of visible inclusions at D12 of adulthood; furthermore, the YFP expression pattern was not significantly affected by the administration of trodusquemine and was constant with age (Figure $3 b$ ). By contrast, PD worms in the absence of trodusquemine were found to have large numbers of inclusions at D12, with the number decreasing significantly (up to 50\%) after treatment with trodusquemine either at the L4 larval stage $(p<0.001$ at D12) or at D5 of adulthood ( $p<0.001$ at D12). Indeed, treatment of the PD worms at the L4 larval stage, prior to the appearance of $\alpha$-synuclein aggregates, significantly reduced the rate of formation of visible $\alpha$-synuclein inclusions as the animals progressed through adult stages (Figure 3c). In addition, initiation of treatment of the PD worms as adults on D5, after $\alpha$-synuclein inclusions had already appeared in the animals, also significantly decreased the subsequent rate of formation of inclusions as the animals aged (Figure 3c).
In an additional series of experiments, the functional behavior of the worms was found to be correlated with the observed effects on inclusion formation. We observed that PD worms treated at the L4 larval stage showed a strongly increased frequency of body bends per time unit $(p<0.001$ at D14), an increased speed of movement $(p<0.001$ at D14), and a decreased rate of paralysis $(p<0.001$ at D14) in comparison to untreated PD worms. Indeed, the behavior of the PD worms treated with $10 \mu \mathrm{M}$ trodusquemine was comparable to that of the healthy control worms (Figure 3d). Accordingly, trodusquemine appears to share similarities in its mode of action with compounds that inhibit the primary nucleation events in protein aggregation such as squalamine. ${ }^{29}$ We observed in addition, however, that PD worms treated at D5 of adulthood also showed a greatly decreased fraction of paralyzed animals $(p<0.001$ at D14) in combination with increased body bends per time unit $(p<0.001$ at D14) and speed of movement ( $p<0.001$ at D14) (Figure 3d). Taken together, these results suggest strongly that trodusquemine has the ability to inhibit secondary nucleation as observed in vitro (Figure 1). 


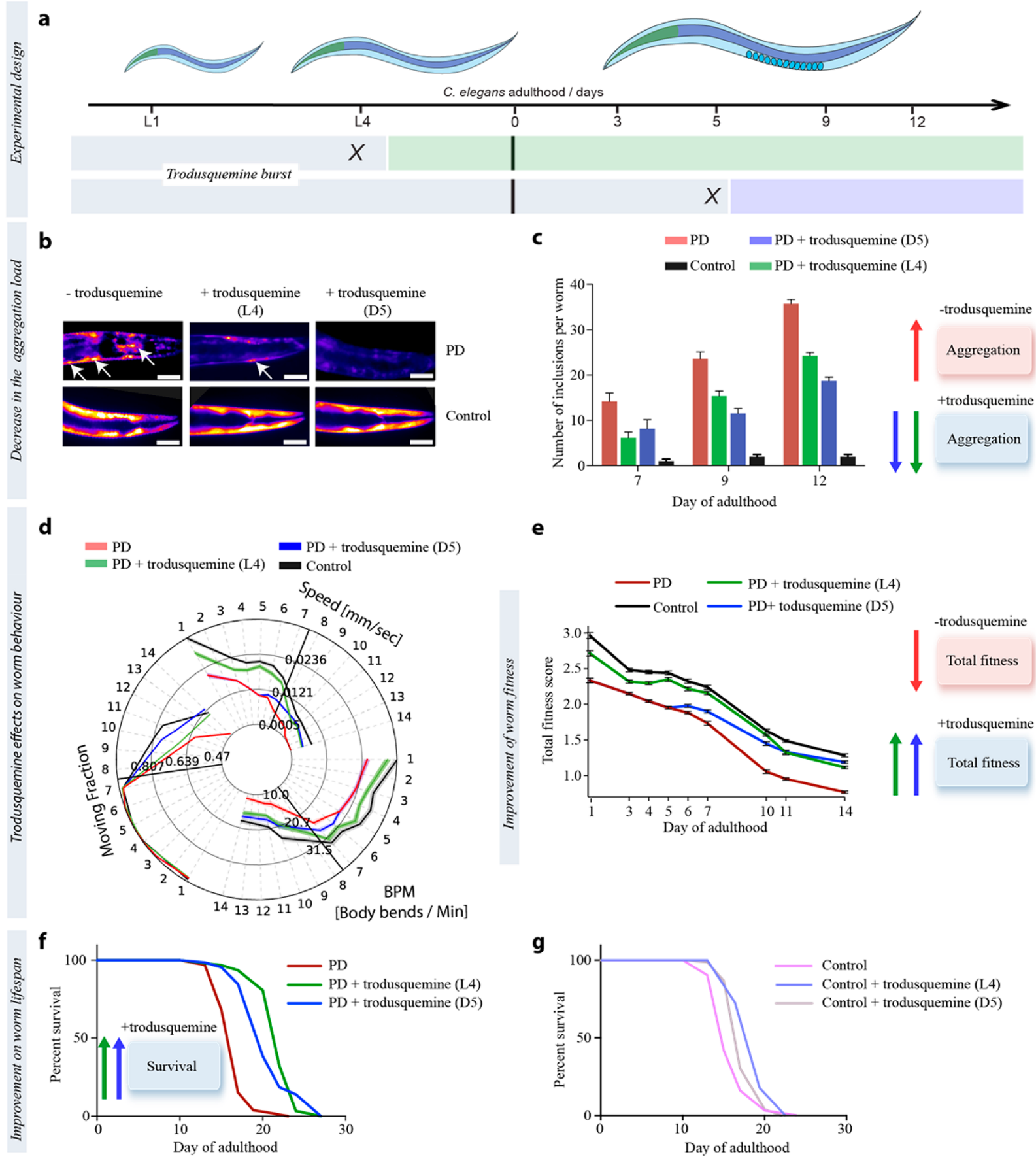

Figure 3. Reduction of the quantity of $\alpha$-synuclein aggregates and improvement of fitness in $C$. elegans PD model through treatment with trodusquemine. (a) Trodusquemine was administered to the PD worms at either the L4 larval stage or D5 of adulthood. (b) Representative images showing the effects of trodusquemine on PD worms expressing $\alpha$-synuclein fused to yellow fluorescent protein (YFP). Worms expressing only YFP in the large muscle cells were used as controls. For every experiment, $N=50$. The images shown are representative of day 12 of adulthood. The scale bar indicates $80 \mu \mathrm{m}$. (c) Effects of trodusquemine on inclusion formation in PD worms at 7, 9, and 12 days of adulthood, respectively. In all panels, the experimental errors refer to SEM. (d) Behavioral map showing the effect of trodusquemine on three phenotypic readouts of worm fitness, i.e. paralysis rate, bends per minute (BPM), and speed of swimming, at the indicated days of adulthood. For every time-point, $N=500$. (e) Total fitness score quantification, ${ }^{29}$ following treatment with trodusquemine. For every time-point, $N=500 .(\mathrm{f}, \mathrm{g})$ Effects following the treatment with trodusquemine on PD (f) and wild type (g) worm survival. For every condition, $N=75$.

Thus, in this animal model of PD, trodusquemine exhibits both prophylactic and therapeutic efficacy with respect to the formation of $\alpha$-synuclein aggregates. By calculating the "total fitness" score, which is a linear combination of the various types of behavior of the worms, including frequency of body bend, speed of movement, and paralysis rate, ${ }^{29}$ the significant increase in the health of $\mathrm{PD}$ worms treated with trodusquemine at L4 $(p<0.001$ at D14) and D5 ( $p<0.001$ at D14) in comparison to untreated PD worms is clearly evident (Figure $3 \mathrm{e}$ ).

In addition to such protective actions on the effects of aggregation, exposure of trodusquemine at either L4 $(p<0.001$ at $\mathrm{D} 20)$ or D5 ( $p<0.001$ at D20) significantly increased the longevity of the PD worms, related to untreated animals (Figure 3f). The lifespan (described here as the age at which there is the $50 \%$ 
mortality of the population) of untreated PD worms was about $17 \pm 1$ days, similar to that of control individuals (Figure 3f). Treatment of the PD worms at L4 extended longevity to $24 \pm 1$ days and at D5 to $20 \pm 1$ days, in each case exceeding the longevity of healthy control animals. Intriguingly, trodusquemine administration also improved the survival of control animals (Figure 3g), although to a lesser extent, suggesting that the effect of trodusquemine on lifespan is enhanced in the presence of tissue injury caused by the accumulation of $\alpha$-synuclein inclusions. Longevity in C. elegans has been studied extensively, and the roles of multiple pathways including insulin/insulin-like growth factor (IGF)-1 signaling, and dietary restriction, have been shown to influence lifespan. ${ }^{52-57}$ Of particular significance in the context of our observations is the report that trodusquemine can stimulate regeneration in various vertebrate tissues and organs following injury, including those of adult mice, through mobilization of stem cells, with no apparent effect on the growth of uninjured tissues. ${ }^{39}$ The electrostatic interactions between trodusquemine and membrane-associated phosphatidylinositol 3,4,5-triphosphate (PIP3), driven by the spermine moiety, could also play an important role in this phenomenon, as the displacement of specific PIP3 binding proteins has been shown to increase fitness and longevity in wild type and PD models in $C$. elegans. ${ }^{58}$ Further experiments will, however, be required to define in detail how trodusquemine extends, in particular, the lifespan of the PD worms and its potential relevance to human subjects.

\section{CONCLUSIONS}

We have shown that the aminosterol trodusquemine inhibits the lipid-induced initiation of aggregation of $\alpha$-synuclein in vitro, whereas it has no detectable effect on the rate of elongation of fibrils. As with the related aminosterol squalamine, trodusquemine inhibits the initiation of aggregation, but we have also found, however, that it inhibits the secondary nucleation of $\alpha$-synuclein, thereby reducing the proliferation of the aggregates. The reduction by trodusquemine of both these nucleation processes will reduce substantially the number of potentially toxic aggregates, providing an explanation for the observation of the substantial protective effects of trodusquemine observed both in cultured cells and in a $C$. elegans model of PD. As with squalamine, ${ }^{29}$ trodusquemine appears to be able to displace $\alpha$-synuclein and its oligomers from the membrane, inhibiting both the lipidinduced initiation of the aggregation process and the ability of the oligomers to disrupt the integrity of membranes. In addition, these molecules may interact directly with the oligomers to reduce their inherent toxicity. Intriguingly, administration of trodusquemine also extends the longevity of the PD worms, beyond even that of control animals and to an extent larger than that observed with squalamine. Taken together, these results suggest that trodusquemine, which can cross the blood-brain barrier, could have multiple benefits in the context of human $\alpha$ synucleinopathies, ranging from an increased understanding of the nature and progression of these conditions to disease modification and tissue regeneration.

\section{MATERIALS AND METHODS}

Reagents. 1,2-Dimyristoyl-sn-glycero-3-phospho-L-serine (sodium salt; DMPS) was purchased from Avanti Polar Lipids (Alabaster, AL, USA). Trodusquemine (as the chloride salt) was synthesized as previously described ${ }^{31}$ and was greater than $97 \%$ pure as evaluated by mass spectrometry. Trodusquemine powder was used immediately after dilution.
Protein Expression and Lipid Preparation. Wild type human $\alpha$-synuclein was recombinantly expressed and purified as described previously. ${ }^{14,15}$ For concentration measurements, we used an extinction coefficient of $5600 \mathrm{M}^{-1} \mathrm{~cm}^{-1}$ at $275 \mathrm{~nm}$. After the final size-exclusion chromatography step ( $20 \mathrm{mM}$ phosphate buffer, $\mathrm{pH} 6.5)$, the protein was snap frozen in liquid nitrogen in the form of $1 \mathrm{~mL}$ aliquots and stored at $-80^{\circ} \mathrm{C}$. These aliquots were used without further treatment, except for the aggregation experiments at low $\mathrm{pH}$ where the $\mathrm{pH}$ was adjusted to the desired value with small volumes of $100 \mathrm{mM} \mathrm{NaOH}$ or $\mathrm{HCl}$. The lipids were dissolved in $20 \mathrm{mM}$ phosphate buffer $\left(\mathrm{NaH}_{2} \mathrm{PO}_{4} /\right.$ $\mathrm{Na}_{2} \mathrm{HPO}_{4}$ ), $\mathrm{pH} 6.5$, and $0.01 \% \mathrm{NaN}_{3}$ and stirred at ca. $45^{\circ} \mathrm{C}$ for $2 \mathrm{~h}$. The solution was then frozen and thawed five times using dry ice and a water bath at $45^{\circ} \mathrm{C}$. The preparation of vesicles was carried out on ice by means of sonication $(3 \times 5 \mathrm{~min}, 50 \%$ cycles, $10 \%$ maximum power on ice) using a Bandelin Sonopuls HD 2070 (Bandelin, Berlin, Germany). After centrifugation, the sizes of the vesicles were checked using dynamic light scattering (Zetasizer Nano ZSP, Malvern Instruments, Malvern, UK) and were shown to consist of a distribution centered on a diameter of $20 \mathrm{~nm}$.

Circular Dichroism (CD) Spectroscopy. Data Acquisition. Samples were prepared as described previously ${ }^{29}$ by incubating $5 \mu \mathrm{M}$ $\alpha$-synuclein with $250 \mu \mathrm{M}$ DMPS vesicles in $20 \mathrm{mM}$ phosphate buffer, $\mathrm{pH} 6.5,0.01 \% \mathrm{NaN}_{3}$, and $0-50 \mu \mathrm{M}$ trodusquemine. Far-UV CD spectra were recorded on a JASCO J-810 instrument (Tokyo, Japan) equipped with a Peltier thermally controlled cuvette holder at $30{ }^{\circ} \mathrm{C}$. Quartz cuvettes with path lengths of $1 \mathrm{~mm}$ were used, and the $\mathrm{CD}$ signal was measured in each case at $222 \mathrm{~nm}$ by averaging 60 individual measurements with a bandwidth of $1 \mathrm{~nm}$, a data pitch of $0.2 \mathrm{~nm}$, a scanning speed of $50 \mathrm{~nm} / \mathrm{min}$, and a response time of $1 \mathrm{~s}$. The signal of the buffer containing DMPS and the different concentrations of trodusquemine was subtracted from that of the protein.

Data Analysis. The concentration of $\alpha$-synuclein bound to DMPS vesicles $\left(\left[\alpha_{\mathrm{b}}\right]\right)$ when $5 \mu \mathrm{M} \alpha$-synuclein was incubated in the presence of $250 \mu \mathrm{M}$ DMPS and increasing concentrations of trodusquemine $([\mathrm{T}])$ was calculated from the $\mathrm{CD}$ signal measured at $222 \mathrm{~nm}$ as described previously. ${ }^{14}$ The change in $\left[\alpha_{\mathrm{b}}\right]$ with increasing $[\mathrm{T}]$ was then analyzed as described previously ${ }^{29}$ using the standard solution of the cubic equation:

$$
\begin{aligned}
K_{\mathrm{D}, \alpha}= & \frac{\left([\mathrm{DMPS}]-L_{\mathrm{T}}\left[\mathrm{T}_{\mathrm{b}}\right]-L_{\alpha}\left[\alpha_{\mathrm{b}}\right]\right)\left([\alpha]-\left[\alpha_{\mathrm{b}}\right]\right)}{L_{\alpha}\left[\alpha_{\mathrm{b}}\right]} \\
{\left[T_{\mathrm{b}}\right]=} & \left([\mathrm{DMPS}]-L_{\alpha}\left[\alpha_{\mathrm{b}}\right]+K_{\mathrm{D}, \mathrm{T}} L_{\mathrm{T}}+L_{\mathrm{T}}[\mathrm{T}]\right. \\
& -\left(4 L_{\mathrm{T}}\left(\left[\alpha_{\mathrm{b}}\right] L_{\alpha}[\mathrm{T}]-[\mathrm{DMPS}][\mathrm{T}]\right)\right. \\
& \left.\left.+\left([\mathrm{DMPS}]-\left[\alpha_{\mathrm{b}}\right] L_{\alpha}+K_{\mathrm{D}, \mathrm{T}} L_{\mathrm{T}}+L_{\mathrm{T}}[\mathrm{T}]\right)^{2}\right)^{-1 / 2}\right) \\
& /\left(2 L_{\mathrm{T}}\right)
\end{aligned}
$$

where $K_{\mathrm{D}, \alpha}$ and $K_{\mathrm{D}, \mathrm{T}}$ are the binding constants of $\alpha$-synuclein and trodusquemine, respectively; $L_{\alpha}$ and $L_{\mathrm{T}}$ are the stoichiometries at which DMPS binds to $\alpha$-synuclein and trodusquemine, i.e., the number of lipid molecules interacting with one molecule of either $\alpha$-synuclein or trodusquemine, respectively; [DMPS], $[\alpha]$, and $[\mathrm{T}]$ are the total concentrations of DMPS, $\alpha$-synuclein, and trodusquemine, respectively; and $\left[\alpha_{\mathrm{b}}\right]$ and $\left[T_{\mathrm{b}}\right]$ are the concentrations of $\alpha$-synuclein and trodusquemine bound to DMPS vesicles, respectively. The best fit is shown in Figure S2 with $K_{\mathrm{D}, \mathrm{T}}=1.62 \times 10^{-8} \mathrm{M}$ and $\left[L_{\mathrm{T}}\right]=5.1$. For further details of the analysis, see the Supporting Information.

Dynamic Light Scattering (DLS) Measurements. Measurements of vesicle size distributions in the absence and presence of the indicated concentrations of trodusquemine were carried out by dynamic light scattering (DLS) experiments using a Zetasizer Nano ZSP Instrument (Malvern Instruments, Malvern, UK) with backscatter detection at a scattering angle of $173^{\circ}$. The concentration of the vesicles was $0.1 \mathrm{mM}$ 
in phosphate buffer (20 mM, pH 6.5) and the experiments were carried out at a temperature of $25^{\circ} \mathrm{C}$.

Aggregation Kinetics in the Presence of Lipid Vesicles. Data Acquisition. $\alpha$-Synuclein was incubated at a concentration of $20-100 \mu \mathrm{M}$ in $20 \mathrm{mM}$ sodium phosphate, $\mathrm{pH} 6.5$, and $0.01 \% \mathrm{NaN}_{3}$, in the presence of $50 \mu \mathrm{M} \mathrm{ThT}, 100 \mu \mathrm{M}$ DMPS vesicles, and increasing concentrations of trodusquemine (0 to $10 \mu \mathrm{M}){ }^{29}$ The stock solution of trodusquemine was prepared by dissolving the molecule in $20 \mathrm{mM}$ phosphate buffer to a final concentration of $100 \mu \mathrm{M}$. The change in the ThT fluorescence signal with time was monitored using a Fluostar Optima or a Polarstar Omega (BMG Labtech, Aylesbury, UK) fluorimeter under quiescent conditions at $30^{\circ} \mathrm{C} .{ }^{29}$ Corning 96 -well plates with half-area (black/clear bottom) nonbinding surfaces (Sigma-Aldrich, St. Louis, MO, USA) were used for each experiment.

Data Analysis. The early times of the aggregation curves of $\alpha$-synuclein in the presence of DMPS and different concentrations of trodusquemine were fitted using the one-step nucleation model described previously ${ }^{14}$ and the following equation:

$$
M(t)=\frac{K_{M} k_{+} m(0)^{n+1} k_{n}[\mathrm{DMPS}] t^{2}}{2\left(K_{M}+m(0)\right) L_{\alpha}}
$$

where $t$ is the time, $M(t)$ is the aggregate mass, $m(0)$ is the free monomer concentration, $K_{M}$ is the saturation constant of the elongation process $(125 \mu \mathrm{M}),{ }^{14} k_{n}$ and $k_{+}$are the rate constants of nucleation and elongation, respectively, and $[\mathrm{DMPS}] / \mathrm{L}_{\alpha}$ is the concentration of proteinbinding sites at the surface of the membrane. First, the early times of the kinetic traces measured for $\alpha$-synuclein in the presence of $100 \mu \mathrm{M}$ DMPS and in the absence of trodusquemine were fitted using eq 3 , with $k_{n} k_{+}$and $n$ being global fitting parameters (see fits in Figure S4a). The global fit yields $n=0.745$ and $k_{n} k_{+}=8.6 \times 10^{-3} \mathrm{M}^{-(n+1)} \cdot \mathrm{s}^{-2}$. We then fixed $n$ to 0.745 and fitted the early times of the data in the presence of trodusquemine using eq 3 , with $k_{n} k_{+}$being the only global fitting parameter (see Figure $\mathrm{S} 4 \mathrm{~b}-\mathrm{f}$ ). This way, we obtain the effective rate of lipidinduced aggregation of $\alpha$-synuclein relative to that in the absence of trodusquemine, at all $\alpha$-synuclein and trodusquemine concentrations (see Figure S5):

$$
r_{\text {eff }}=\frac{k_{n} k_{+}^{\prime}}{k_{n} k_{+}}
$$

where $k_{n} k_{+}^{\prime}$ is the product of the nucleation and elongation rate constants at a given [trodusquemine $] /[\alpha$-synuclein $]$ ratio. If trodusquemine were to inhibit $\alpha$-synuclein lipid-induced aggregation via the same mechanism as that reported previously for squalamine ${ }^{29}$ and $\beta$-synuclein, ${ }^{14,41}$ the effective rate should scale with a power of the coverage, i.e., $r_{\text {eff }}=\theta_{\alpha}^{n_{\mathrm{b}}}$, where $\theta_{\alpha}$ is the fractional coverage of a lipid vesicle in $\alpha$-synuclein and $n_{\mathrm{b}}$ is the reaction order of lipid-induced aggregation with respect to $\theta_{\alpha}$. In the absence of trodusquemine, $\theta_{\alpha}=1$, as we are in a regime where the vesicles are saturated. We determined $\theta_{\alpha}$ for the different [trodusquemine $] /[\alpha$-synuclein $]$ ratios used in our study using a simplified competitive binding model with the values of $K_{\mathrm{D}, \mathrm{T}}$ and $L_{\mathrm{T}}$ determined in this study and $K_{\mathrm{D}, \alpha}$ and $L_{\alpha}$ determined previously, ${ }^{14}$ using $n_{\mathrm{b}}=5.5$, as determined previously, ${ }^{29,41}$ and the following equations:

$$
\begin{aligned}
& \theta_{\alpha}=\frac{\left[\alpha_{\mathrm{b}}\right] L_{\alpha}}{[\mathrm{DMPS}]} \\
& {\left[\alpha_{\mathrm{b}}\right]=}\left([\mathrm{DMPS}] L_{\alpha} \kappa-[\mathrm{DMPS}] L_{\mathrm{T}}-[\alpha] L_{\alpha} L_{\mathrm{T}}-L_{\alpha} L_{\mathrm{T}}[\mathrm{T}] \kappa\right) \\
& /\left(2\left(L_{\alpha}^{2} \kappa-L_{\alpha} L_{\mathrm{T}}\right)\right)+\left(\left(4[\alpha][\mathrm{DMPS}] L_{\mathrm{T}}\left(L_{\alpha}^{2} \kappa-L_{\alpha} L_{\mathrm{T}}\right)\right.\right. \\
&+\left([\mathrm{DMPS}] L_{\mathrm{T}}+[\alpha] L_{\alpha} L_{\mathrm{T}}-[\mathrm{DMPS}] L_{\alpha} \kappa\right. \\
&\left.\left.\left.+L_{\alpha} L_{\mathrm{T}}[\mathrm{T}] \kappa\right)^{2}\right)^{-1 / 2}\right) /\left(2\left(L_{\alpha}^{2} \kappa-L_{\alpha} L_{\mathrm{T}}\right)\right)
\end{aligned}
$$

where $\kappa=K_{\mathrm{D}, \alpha} / K_{\mathrm{D}, \mathrm{T}}$.

We found that the values of $r_{\text {eff }}$ obtained at different [trodusquemine $] /[\alpha$-synuclein $]$ ratios do not scale simply as $\theta_{\alpha}^{n_{b}}$, with the coverage $\theta_{\alpha}$ calculated using eqs 5 and 6 at the corresponding ratios, suggesting that trodusquemine inhibits $\alpha$-synuclein lipid-induced aggregation via a more complex mechanism than that described for squalamine (see Figure S5), perhaps resulting from a direct interaction with the oligomeric species resulting in an inhibition of their inherent toxicity.

Seed Fibril Formation. Seed fibrils were produced as previously described. ${ }^{15,41}$ Briefly, we incubated $500 \mu \mathrm{L}$ solutions of $\alpha$-synuclein at concentrations between 500 and $800 \mu \mathrm{M}$ in $20 \mathrm{mM}$ phosphate buffer at $\mathrm{pH} 6.5$ for $48-72 \mathrm{~h}$ at ca. $40^{\circ} \mathrm{C}$ with maximal stirring with a Teflon bar on an RCT Basic Heat Plate (IKA, Staufen, Germany). The fibrils were divided into aliquots, frozen in liquid $\mathrm{N}_{2}$, and stored at $-80{ }^{\circ} \mathrm{C}$ until required. For aggregation experiments, the seed fibrils were diluted to $200 \mu \mathrm{M}$ monomer equivalents into the specific buffer to be used in the experiment and sonicated three times for $10 \mathrm{~s}$ using a Bandelin Sonopuls HD 2070 probe sonicator (Bandelin, Berlin, Germany), using 10\% maximum power and $30 \%$ cycles.

Seeded Aggregation Kinetics. The seeded experiments were performed as described previously. ${ }^{15,41}$ Briefly, to probe fibril elongation, preformed seed fibrils ( $5 \mu \mathrm{M}$ monomer equivalents) were added to solutions of monomeric $\alpha$-synuclein $(20-100 \mu \mathrm{M})$ in $20 \mathrm{mM}$ phosphate buffer ( $\mathrm{pH} 6.5$ ) with $50 \mu \mathrm{M} \mathrm{ThT}$, under quiescent conditions, at $37^{\circ} \mathrm{C}$. For experiments to probe the influence on secondary nucleation, seeds (50 $\mathrm{nM}$ monomer equivalents) were added to monomeric $\alpha$-synuclein $(60-100 \mu \mathrm{M})$ in $20 \mathrm{mM}$ phosphate buffer $(\mathrm{pH} 4.8)$ and in the presence of $50 \mu \mathrm{M} \mathrm{ThT}$, under quiescent conditions, also at $37^{\circ} \mathrm{C}$. The increase in ThT fluorescence was monitored in low binding, clear-bottomed halfarea Corning 96 well plates (Sigma-Aldrich, St. Louis, MO, USA) that were sealed with tape, and using either a Fluostar Optima, Polarstar Omega (BMG Labtech, Aylesbury, UK), or M1000 (Tecan Group Ltd., Männedorf, Switzerland) fluorescence plate-reader in bottom reading mode. All experiments were performed under quiescent conditions (i.e., without shaking).

Data Analysis. Data were analyzed as previously described. ${ }^{15,41}$

Mass Spectrometry. Experiments were carried out as previously described. ${ }^{29}$ Briefly, fibrils of $\alpha$-synuclein at a concentration of $10 \mu \mathrm{M}$ (monomer equivalents) were incubated with $10 \mu \mathrm{M}$ trodusquemine in $20 \mathrm{mM}$ Tris, $\mathrm{pH} 7.4$, and $100 \mathrm{mM} \mathrm{NaCl}$ overnight under quiescent conditions at RT. The samples were then centrifuged at $100,000 \mathrm{~g}$ for $30 \mathrm{~min}$ and the supernatant then removed for analysis. Samples for the analysis by mass spectrometry were prepared as described, ${ }^{29}$ and the experiments were run using a Waters Xevo G2-S QTOF sprectrometer (Waters Corporation, MA, USA).

Nuclear Magnetic Resonance (NMR) Spectroscopy. $\mathrm{H}_{2} \mathrm{O}$ was removed from trodusquemine by three cycles of lyophilization, and the compound was resolvated in $100 \% \mathrm{D}_{2} \mathrm{O}$ (Sigma-Aldrich). It was then diluted into $20 \mathrm{mM}$ phosphate buffer, $\mathrm{pH} 6.5$, in $100 \% \mathrm{D}_{2} \mathrm{O}$ to a final concentration of $10 \mu \mathrm{M}$. All NMR measurements were performed at $30{ }^{\circ} \mathrm{C}$ on a Bruker AVANCE-500 spectrometer, operated at a ${ }^{1} \mathrm{H}$ frequency of $500.13 \mathrm{MHz}$, equipped with a cryogenic probe. Measurements of diffusion coefficients were performed using $2 \mathrm{D}{ }^{1} \mathrm{H}$ diffusion ordered spectroscopy (DOSY) experiments. ${ }^{59}$ These spectra were acquired using the standard "ledbpgppr2s" Bruker pulse program, using a bipolar gradient pulse pair-stimulated echo sequence incorporating a longitudinal eddy current delay, ${ }^{60}$ with a diffusion time $(\Delta)$ of $100 \mathrm{~ms}$ and a gradient pulse length $(\delta)$ of $3 \mathrm{~ms}$ and increasing the gradient strength between $4.8<g<38.5 \mathrm{G} \mathrm{cm}^{-1}$. The values of $\Delta$ and $\delta$ were chosen based on measurements of $1.5 \mathrm{mM}$ trodusquemine in water, in which it is very soluble. To remove the signal from residual $\mathrm{H}_{2} \mathrm{O}$ in the sample, presaturation was used. Data for $1.5 \mathrm{mM}$ trodusquemine in $100 \% \mathrm{D}_{2} \mathrm{O}$ were collected with eight scans and 16 gradient steps, while data for $10 \mu \mathrm{M}$ trodusquemine in $20 \mathrm{mM}$ phosphate buffer were collected with 400 scans and 12 gradient steps. Individual rows of the pseudo-2D diffusion data were phased and baseline corrected. DOSY spectra were processed using the TopSpin 2.1 software (Bruker). The diffusion dimension was generated using the intensities $(I)$ of resolved peaks between 3.0 and -0.5 ppm according to the Stejskal-Tanner equation $^{61}$

$$
\frac{I}{I_{0}}=\mathrm{e}^{-\gamma^{2} g^{2} \delta^{2} D\left(\Delta-\frac{\delta}{3}\right)}
$$

where $\gamma$ is the gyromagnetic ratio, and using the DynamicsCenter 2.5.3 software (Bruker). $1 \mathrm{D}{ }^{1} \mathrm{H}$ NMR spectra of $10 \mu \mathrm{M}$ trodusquemine 
alone in $20 \mathrm{mM}$ phosphate buffer, $\mathrm{pH} 6.5$ and $30{ }^{\circ} \mathrm{C}$, made up in $100 \% \mathrm{D}_{2} \mathrm{O}$ in the presence or absence of equimolar 4,4-dimethyl-4silapentane-1-sulfonic acid (DSS, Sigma-Aldrich) as an internal concentration standard. These spectra were acquired using the standard "noesyprld" Bruker pulse program with presaturation to remove the signal from residual $\mathrm{H}_{2} \mathrm{O}$ in the sample. Spectra were processed using TopSpin 2.1 .

ThT Binding to Trodusquemine. Preformed fibrils of $\alpha$-synuclein $(5 \mu \mathrm{M})$ were added to monomeric $\alpha$-synuclein at a concentration of $100 \mu \mathrm{M}$ and incubated in 96-well half-area, low-binding polyethylene glycol coating plate (Corning 3881, Kennebuck ME, USA) at $37^{\circ} \mathrm{C}$ in $20 \mathrm{mM}$ phosphate buffer ( $\mathrm{pH}$ 6.5) under quiescent conditions for $24 \mathrm{~h}$. Then, the resulting longer fibrils were diluted to $50 \mu \mathrm{M} \alpha$-synuclein with $50 \mu \mathrm{M}$ ThT and the absence or presence of the indicated concentration of trodusquemine in $20 \mathrm{mM}$ phosphate buffer ( $\mathrm{pH} 6.5$ ) and incubated at $37^{\circ} \mathrm{C}$ for $12 \mathrm{~h}$. The fluorescence intensity of the different samples was measured using a plate reader (Fluostar Optima, BMG Labtech, Ortenberg, Germany)

Preparation of Oligomers. Samples of $\alpha$-synuclein type B* oligomers were prepared as previously described. ${ }^{47}$ Briefly, monomeric $\alpha$-synuclein was lyophilized in Milli-Q water and subsequently resuspended in PBS, pH 7.4, to give a final concentration of ca. $800 \mu \mathrm{M}$ $\left(12 \mathrm{mg} \mathrm{mL}^{-1}\right)$. The resulting solutions were passed through a $0.22 \mu \mathrm{m}$ cutoff filter before incubation at $37^{\circ} \mathrm{C}$ for $20-24 \mathrm{~h}$ under quiescent conditions. ${ }^{47}$ Very small amounts of fibrillar species formed during this time were removed by ultracentrifugation for $1 \mathrm{~h}$ at $90000 \mathrm{rpm}$ (using a TLA-120.2 Beckman rotor, $288000 \mathrm{~g}$ ). Excess monomeric protein and any small oligomeric species were then removed by multiple cycles of filtration using $100 \mathrm{kDa}$ cutoff membranes. The final concentration of the prepared oligomers was estimated using $\varepsilon_{275}=$ $5600 \mathrm{M}^{-1} \mathrm{~cm}^{-1}$.

Neuroblastoma Cell Cultures. Human SH-SY5Y neuroblastoma cells (A.T.C.C., Manassas, VA, USA) were cultured in DMEM, F-12 HAM with $25 \mathrm{mM}$ HEPES, and $\mathrm{NaHCO}_{3}$ (1:1) and supplemented with $10 \%$ FBS, $1 \mathrm{mM}$ glutamine, and $1.0 \%$ antibiotics. Cell cultures were maintained in a $5 \% \mathrm{CO}_{2}$ humidified atmosphere at $37{ }^{\circ} \mathrm{C}$ and grown until they reached $80 \%$ confluence for a maximum of 20 passages. ${ }^{62}$

MTT Reduction Assay. $\alpha$-Synuclein oligomers ( $0.3 \mu \mathrm{M}$ monomer equivalents) were incubated without or with increasing concentrations $(0.03,0.1$, and 0.3$)$ of trodusquemine for $1 \mathrm{~h}$ at $37^{\circ} \mathrm{C}$ under shaking conditions and then added to the cell culture medium of SH-SY5Y cells seeded in 96-well plates for $24 \mathrm{~h}$. The 3-(4,5-dimethylthiazol-2-yl)-2,5diphenyltetrazolium bromide (MTT) reduction assay was performed as previously described. ${ }^{63}$

Measurement of Intracellular ROS. SH-SYSY cells were seeded on glass coverslips and treated for $15 \mathrm{~min}$ with $\alpha$-synuclein oligomers $(0.3 \mu \mathrm{M})$ and increasing concentrations $(0.03,0.3$, and $3 \mu \mathrm{M})$ of trodusquemine. After incubation, the cells were washed with PBS and loaded with $10 \mu \mathrm{M} \mathrm{2} 2^{\prime}, 7^{\prime}$-dichlorodihydrofluorescein diacetate (CM- $\mathrm{H}_{2}$ DCFDA; Life Technologies, CA, USA) as previously described. ${ }^{62}$ The fluorescence of the cells was then analyzed by means of a TCS SP5 scanning confocal microscopy system (Leica Microsystems, Mannheim, Germany) equipped with an argon laser source, using the $488 \mathrm{~nm}$ excitation line. A series of $1.0-\mu \mathrm{m}$-thick optical sections $(1024 \times$ 1024 pixels) was taken through the cells for each sample using a Leica Plan Apo 63 oil immersion objective.

Oligomer Binding to Cellular Membranes. SH-SY5Y cells were seeded on glass coverslips and treated for $15 \mathrm{~min}$ with $\alpha$-synuclein oligomers $(0.3 \mu \mathrm{M})$ and increasing concentrations $(0.03,0.3$, and $3 \mu \mathrm{M})$ of trodusquemine. After incubation, the cells were washed with PBS and counterstained with $5.0 \mu \mathrm{g} / \mathrm{mL}$ Alexa Fluor 633-conjugated wheat germ agglutinin (Life Technologies, CA, USA) to label fluorescently the cellular membrane. ${ }^{29}$ After washing with PBS, the cells were fixed in $2 \%(\mathrm{w} / \mathrm{v})$ buffered paraformaldehyde for $10 \mathrm{~min}$ at RT $\left(20^{\circ} \mathrm{C}\right)$. The presence of oligomers was detected with 1:250 diluted rabbit polyclonal anti- $\alpha$-synuclein antibodies (Abcam, Cambridge, UK) and subsequently with 1:1000 diluted Alexa Fluor 488-conjugated antirabbit secondary antibodies (Life Technologies, CA, USA). Fluorescence emission was detected after double excitation at 488 and $633 \mathrm{~nm}$ by the scanning confocal microscopy system described above, and three apical sections were projected as a single composite image by superimposition.

C. elegans Media. Standard conditions were used for the propagation of C. elegans. ${ }^{64}$ Briefly, the animals were synchronized by hypochlorite bleaching, hatched overnight in $\mathrm{M} 9\left(3 \mathrm{~g} / \mathrm{L} \mathrm{KH}_{2} \mathrm{PO}_{4}, 6 \mathrm{~g} / \mathrm{L} \mathrm{Na}_{2} \mathrm{HPO}_{4}\right.$, $5 \mathrm{~g} / \mathrm{L} \mathrm{NaCl}, 1 \mu \mathrm{M} \mathrm{MgSO}_{4}$ ) buffer, and subsequently cultured at $20^{\circ} \mathrm{C}$ on nematode growth medium (NGM) plates $(1 \mathrm{mM} \mathrm{CaCl}, 1 \mathrm{mM}$ $\mathrm{MgSO}_{4}, 5 \mu \mathrm{g} / \mathrm{mL}$ cholesterol, $250 \mu \mathrm{M} \mathrm{KH}_{2} \mathrm{PO}_{4}, \mathrm{pH} 6,17 \mathrm{~g} / \mathrm{L}$ Agar, $3 \mathrm{~g} / \mathrm{L} \mathrm{NaCl}, 7.5 \mathrm{~g} / \mathrm{L}$ casein) seeded with the E. coli strain OP50. Saturated cultures of OP50 were grown by inoculating $50 \mathrm{~mL}$ of LB medium $(10 \mathrm{~g} / \mathrm{L}$ tryptone, $10 \mathrm{~g} / \mathrm{L} \mathrm{NaCl}, 5 \mathrm{~g} / \mathrm{L}$ yeast extract) with OP50 cells and incubating the culture for $16 \mathrm{~h}$ at $37^{\circ} \mathrm{C}$. NGM plates were seeded with bacteria by adding $350 \mu \mathrm{L}$ of saturated OP50 cells to each plate and leaving the plates at $20^{\circ} \mathrm{C}$ for $2-3$ days. On day 3 after synchronization, the animals were placed on NGM plates containing $75 \mu \mathrm{M}$ 5-fluoro-2' deoxyuridine (FUDR).

Strains. The following strains were used: zgIs15 $[\mathrm{P}($ unc-54):: $\alpha$ syn::YFP]IV (OW40), in which $\alpha$-synuclein fused to YFP relocates to inclusions, which are visible as early as day 2 after hatching and increase in number and size during the aging of the animals, up to late adulthood (day 17), ${ }^{29,65}$ and rmIs126 [P(unc-54)Q0::YFP]V (OW450). In OW450, YFP alone is expressed and remains diffusely localized throughout aging. ${ }^{29,65}$

Trodusquemine-coated plates. Plates were prepared as previously described. ${ }^{29}$ Briefly, aliquots of NGM media were autoclaved, poured, and seeded with $350 \mu \mathrm{L}$ of OP50 culture and grown overnight at RT. After incubating for up to 3 days at RT, aliquots of trodusquemine dissolved in water at different concentrations were added. NGM plates containing FUDR ( $75 \mu \mathrm{M}$, unless stated otherwise) were seeded with aliquots of the trodusquemine dissolved in water, at the appropriate concentration. The plates were then placed in a laminar flow hood at RT to dry, and the worms were transferred to plates coated with trodusquemine at larval stage L4.

Automated Motility Assay. All C. elegans populations were cultured at $20{ }^{\circ} \mathrm{C}$ and developmentally synchronized from a $4 \mathrm{~h}$ egglay. At $64-72 \mathrm{~h}$ post-egg-lay (time zero), individuals were transferred to FUDR plates, and body movements were assessed over the times indicated. At different ages, the animals were washed off the plates with M9 buffer and spread over an OP-50 unseeded $6 \mathrm{~cm}$ plate, after which their movements were recorded at $30 \mathrm{fps}$ using a recently developed microscopic procedure for $30 \mathrm{~s}$ or $1 \mathrm{~min}^{29} \mathrm{Up}$ to 200 animals were counted in each experiment unless otherwise stated. One experiment that is representative of the three measured in each series of experiments is shown, and videos were analyzed using a custom-made tracking code. $^{29,66}$

Lifespan Assays. Lifespan analysis was carried out as previously described. ${ }^{67}$ On day 3 after synchronization, the animals were placed on NGM plates containing FUDR. On L4 or D5, they were manually transferred to plates seeded with $10 \mu \mathrm{M}$ trodusquemine. Experiments were performed at $20^{\circ} \mathrm{C}$. Lifespan experiments were performed with 75 animals per condition. At each time point, the number of surviving animals, determined by movement and response to nose touch, was counted. Animals that crawled out of the plates during the assay were excluded. Three independent experiments were carried out in each case, and one representative is shown. Analysis was performed using GraphPad Prism (GraphPad Software).

Quantification of Inclusions. Individual animals were mounted on $2 \%$ agarose pads, containing $40 \mathrm{mM} \mathrm{NaN}_{3}$ as an anesthetic, on glass microscope slides for imaging. ${ }^{29}$ Only the frontal region of the worms was considered. ${ }^{29,65}$ The numbers of inclusions in each animal were quantified using a Leica MZ16 FA fluorescence dissection stereomicroscope (Leica Microsystems, Wetzlar, Germany) at a nominal magnification of $20 \times$ or $40 \times$, and images were acquired using an Evolve 512 Delta EMCCD Camera, with high quantum efficiency (Photometrics, Tucson, AZ, USA). Measurements on inclusions were performed using ImageJ software. ${ }^{29}$ All experiments were carried out in triplicate, and the data from one representative experiment are shown in the figure. The Student's $t$ test was used to calculate $p$ values, and all tests were two-tailed unpaired unless otherwise stated. At least 50 animals were examined per condition, unless stated otherwise. ${ }^{29}$ 


\section{ASSOCIATED CONTENT}

\section{S Supporting Information}

The Supporting Information is available free of charge on the ACS Publications website at DOI: 10.1021/acschembio.8b00466.

Experimental procedures and characterization including the structure of trodusquemine and sequence of $\alpha$-synuclein, dynamic light scattering (DLS), circular dichroism (CD), lipid induced aggregation, global analysis of kinetic traces, effective rate of $\alpha$-synuclein lipid-induced aggregation, fibril elongation and relative rates, fibril amplification and relative rates, mass spectrometry, ThT binding, nuclear magnetic resonance (NMR) (PDF)

\section{AUTHOR INFORMATION}

\section{Corresponding Authors}

*E-mail: fabrizio.chiti@unifi.it.

*E-mail:maz5@georgetown.edu.

*E-mail:mv245@cam.ac.uk.

*E-mail: cmd44@cam.ac.uk.

\section{ORCID}

Michele Perni: 0000-0001-7593-8376

Patrick Flagmeier: 0000-0002-1204-5340

Francesco A. Aprile: 0000-0002-5040-4420

Céline Galvagnion: 0000-0001-9753-3310

Gabriella T. Heller: 0000-0002-5672-0467

Georg Meisl: 0000-0002-6562-7715

Janet R. Kumita: 0000-0002-3887-4964

Pavan K. Challa: 0000-0002-0863-381X

Nunilo Cremades: 0000-0002-9138-6687

Tuomas P. J. Knowles: 0000-0002-7879-0140

Fabrizio Chiti: 0000-0002-1330-1289

Michele Vendruscolo: 0000-0002-3616-1610

Christopher M. Dobson: 0000-0002-5445-680X

\section{Author Contributions}

${ }^{+}$These authors made equal contributions

\section{Author Contributions}

M.P., P.F., R.L., F.A., C.G., T.P.J.K., D.B., M.Z., M.V., F.C., C.C., and C.M.D. were involved in the design of the study. M.P. and R.L. performed the C. elegans experiments. P.F. and C.G. carried out the in vitro experiments. P.F., C.G., and G.M. analyzed the kinetic data of $\alpha$-synuclein aggregation. R.C. carried out the cell experiments. G.T.H. performed the NMR experiments. S.W.C. purified the $\alpha$-synuclein oligomers. M.P., P.F., R.L., M.Z., M.V., F.C., and C.M.D. wrote the paper, and all the authors were involved in the analysis of the data and editing of the paper.

\section{Notes}

The authors declare the following competing financial interest(s): M.Z. and D.B. own patents for the use of Trodusquemine in the treatment of Parkinson's disease.

\section{ACKNOWLEDGMENTS}

This work was supported by the Boehringer Ingelheim Fonds (P.F.), the Studienstiftung des Deutschen Volkes (P.F.), Gates Cambridge Scholarships (R.L. and G.T.H) and a St. John's College Benefactors' Scholarship (R.L.), the UK Biotechnology and Biochemical Sciences Research Council (M.V. and C.M.D.), a Senior Research Fellowship award from the Alzheimer's Society, UK, grant number (317, AS-SF-16-003) (F.A.A.), the Wellcome Trust (C.M.D., M.V., and T.P.J.K.), the Frances and Augustus Newman Foundation (T.P.J.K.), the Regione Toscana-
FAS Salute-Supremal project (R.C., C.C., and F.C.), a Marie Skłodowska-Curie Actions-Individual Fellowship (C.G.), Sidney Sussex College Cambridge (G.M.), the Spanish GovernmentMINECO (N.C.), and by the Cambridge Centre for Misfolding Diseases (M.P., P.F., R.L., F.A.A., C.G., G.T.H., S.W.C., J.R.K., T.P.J.K., M.V., and C.M.D). The authors would like to thank E. Klimont for her assistance with the expression and purification of $\alpha$-synuclein. We also would like to thank N. Fernando and $S$. Casford for assistance with the C. elegans experiments. We acknowledge the NMR Service at the Chemistry Department of the University of Cambridge for helpful discussions, particularly P. Grice and D. Howe and the UK EPSRC for Core Capabilities funding (EP/K039520/1).

\section{REFERENCES}

(1) Bodner, C. R., Dobson, C. M., and Bax, A. (2009) Multiple tight phospholipid-binding modes of alpha-synuclein revealed by solution NMR spectroscopy. J. Mol. Biol. 390, 775-790.

(2) Wilhelm, B. G., Mandad, S., Truckenbrodt, S., et al. (2014) Composition of isolated synaptic boutons reveals the amounts of vesicle trafficking proteins. Science 344, 1023-1028.

(3) Maroteaux, L., Campanelli, J. T., and Scheller, R. H. (1988) Synuclein: a neuron-specific protein localized to the nucleus and presynaptic nerve terminal. J. Neurosci. 8, 2804-2815.

(4) Takeda, A., Mallory, M., Sundsmo, M., Honer, W., Hansen, L., and Masliah, E. (1998) Abnormal accumulation of NACP/alpha-synuclein in neurodegenerative disorders. Am. J. Pathol. 152, 367-372.

(5) Spillantini, M. G., Crowther, R. A., Jakes, R., Hasegawa, M., and Goedert, M. (1998) alpha-Synuclein in filamentous inclusions of Lewy bodies from Parkinson's disease and dementia with lewy bodies. Proc. Natl. Acad. Sci. U. S. A. 95, 6469-6473.

(6) Dettmer, U., Selkoe, D., and Bartels, T. (2016) New insights into cellular $\alpha$-synuclein homeostasis in health and disease. Curr. Opin. Neurobiol. 36, 15-22.

(7) Dawson, T. M., and Dawson, V. L. (2003) Molecular pathways of neurodegeneration in Parkinson's disease. Science 302, 819-822.

(8) Knowles, T. P. J., Vendruscolo, M., and Dobson, C. M. (2014) The amyloid state and its association with protein misfolding diseases. Nat. Rev. Mol. Cell Biol. 15, 384-396.

(9) Chiti, F., and Dobson, C. M. (2006) Protein misfolding, functional amyloid, and human disease. Annu. Rev. Biochem. 75, 333-366.

(10) Lee, V. M. Y., and Trojanowski, J. Q. (2006) Mechanisms of Parkinson's disease linked to pathological alpha-synuclein: new targets for drug discovery. Neuron 52, 33-38.

(11) Tong, J., Wong, H., Guttman, M., Ang, L. C., Forno, L. S., Shimadzu, M., Rajput, A. H., Muenter, M. D., Kish, S. J., Hornykiewicz, O., and Furukawa, Y. (2010) Brain $\alpha$-synuclein accumulation in multiple system atrophy, Parkinson's disease and progressive supranuclear palsy: a comparative investigation. Brain 133, 172-188.

(12) Chiti, F., and Dobson, C. M. (2017) Protein misfolding, amyloid formation, and human disease: a summary of progress over the last decade. Annu. Rev. Biochem. 86, 27-68.

(13) Buell, A. K., Galvagnion, C., Gaspar, R., Sparr, E., Vendruscolo, M., Knowles, T. P. J., Linse, S., and Dobson, C. M. (2014) Solution conditions determine the relative importance of nucleation and growth processes in $\alpha$-synuclein aggregation. Proc. Natl. Acad. Sci. U. S. A. 111, $7671-7676$

(14) Galvagnion, C., Buell, A. K., Meisl, G., Michaels, T. C. T., Vendruscolo, M., Knowles, T. P. J., and Dobson, C. M. (2015) Lipid vesicles trigger $\alpha$-synuclein aggregation by stimulating primary nucleation. Nat. Chem. Biol. 11, 229-234.

(15) Flagmeier, P., Meisl, G., Vendruscolo, M., Knowles, T. P. J., Dobson, C. M., Buell, A. K., and Galvagnion, C. (2016) Mutations associated with familial Parkinson's disease alter the initiation and amplification steps of $\alpha$-synuclein aggregation. Proc. Natl. Acad. Sci. U.S. A. 113, 10328-10333. 
(16) Fink, A. L. (2006) The aggregation and fibrillation of alphasynuclein. Acc. Chem. Res. 39, 628-634.

(17) Munishkina, L. A., Phelan, C., Uversky, V. N., and Fink, A. L. (2003) Conformational behavior and aggregation of alpha-synuclein in organic solvents: modeling the effects of membranes. Biochemistry 42, $2720-2730$.

(18) Munishkina, L. A., Henriques, J., Uversky, V. N., and Fink, A. L. (2004) Role of protein-water interactions and electrostatics in alphasynuclein fibril formation. Biochemistry 43, 3289-3300.

(19) Uversky, V. N., Li, J., Souillac, P., Millett, I. S., Doniach, S., Jakes, R., Goedert, M., and Fink, A. L. (2002) Biophysical properties of the synucleins and their propensities to fibrillate: inhibition of alphasynuclein assembly by beta- and gamma-synucleins. J. Biol. Chem. 277, 11970-11978.

(20) Uversky, V. N., Li, J., and Fink, A. L. (2001) Evidence for a partially folded intermediate in alpha-synuclein fibril formation. J. Biol. Chem. 276, 10737-10744.

(21) Heise, H., Hoyer, W., Becker, S., Andronesi, O. C., Riedel, D., and Baldus, M. (2005) Molecular-level secondary structure, polymorphism, and dynamics of full-length $\alpha$-synuclein fibrils studied by solid-state NMR. Proc. Natl. Acad. Sci. U. S. A. 102 (44), 15871-6.

(22) Vilar, M., Chou, H.-T., Lührs, T., Maji, S. K., Riek-Loher, D., Verel, R., Manning, G., Stahlberg, H., and Riek, R. (2008) The fold of alpha-synuclein fibrils. Proc. Natl. Acad. Sci. U. S. A. 105, 8637-8642.

(23) Comellas, G., Lemkau, L. R., Nieuwkoop, A. J., Kloepper, K. D., Ladror, D. T., Ebisu, R., Woods, W. S., Lipton, A. S., George, J. M., and Rienstra, C. M. (2011) Structured regions of $\alpha$-synuclein fibrils include the early-onset Parkinson's disease mutation sites. J. Mol. Biol. 411, 881895 .

(24) Gath, J., Bousset, L., Habenstein, B., Melki, R., Böckmann, A., and Meier, B. H. (2014) Unlike twins: an NMR comparison of two $\alpha$ synuclein polymorphs featuring different toxicity. PLoS One 9, e90659e90659.

(25) Gath, J., Bousset, L., Habenstein, B., Melki, R., Meier, B. H., and Böckmann, A. (2014) Yet another polymorph of $\alpha$-synuclein: solid-state sequential assignments. Biomol. NMR Assignments 8, 395-404.

(26) Tuttle, M. D., Comellas, G., Nieuwkoop, A. J., Covell, D. J., Berthold, D. A., Kloepper, K. D., Courtney, J. M., Kim, J. K., Barclay, A. M., Kendall, A., Wan, W., Stubbs, G., Schwieters, C. D., Lee, V. M. Y., George, J. M., and Rienstra, C. M. (2016) Solid-state NMR structure of a pathogenic fibril of full-length human $\alpha$-synuclein. Nat. Struct. Mol. Biol. $23,409-415$.

(27) Tóth, G., Gardai, S. J., Zago, W., Bertoncini, C. W., Cremades, N., Roy, S. L., Tambe, M. A., Rochet, J.-C., Galvagnion, C., Skibinski, G., Finkbeiner, S., Bova, M., Regnstrom, K., Chiou, S.-S., Johnston, J., Callaway, K., Anderson, J. P., Jobling, M. F., Buell, A. K., Yednock, T. A., Knowles, T. P. J., Vendruscolo, M., Christodoulou, J., Dobson, C. M., Schenk, D., and McConlogue, L. (2014) Targeting the intrinsically disordered structural ensemble of $\alpha$-synuclein by small molecules as a potential therapeutic strategy for Parkinson's disease. PLoS One 9, e87133-11.

(28) Kakish, J., Lee, D., and Lee, J. S. (2015) Drugs which bind to alpha-synuclein. Neuroprotective or neurotoxic? ACS Chem. Neurosci. 6 (12), 1930-40.

(29) Perni, M., Galvagnion, C., Maltsev, A., Meisl, G., Muller, M. B. D., Challa, P. K., Kirkegaard, J. B., Flagmeier, P., Cohen, S. I. A., Cascella, R., Chen, S. W., Limbocker, R., Sormanni, P., Heller, G. T., Aprile, F. A., Cremades, N., Cecchi, C., Chiti, F., Nollen, E. A. A., Knowles, T. P. J., Vendruscolo, M., Bax, A., Zasloff, M., and Dobson, C. M. (2017) A natural product inhibits the initiation of $\alpha$-synuclein aggregation and suppresses its toxicity. Proc. Natl. Acad. Sci. U. S. A. 114 (6), E1009E1017.

(30) Galvagnion, C., Brown, J. W. P., Ouberai, M. M., Flagmeier, P., Vendruscolo, M., Buell, A. K., Sparr, E., and Dobson, C. M. (2016) Chemical properties of lipids strongly affect the kinetics of the membrane-induced aggregation of $\alpha$-synuclein. Proc. Natl. Acad. Sci. U. S. A. 113, 7065-7070.

(31) Zasloff, M., Williams, J. I., Chen, Q., Anderson, M., Maeder, T., Holroyd, K., Jones, S., Kinney, W., Cheshire, K., and McLane, M. (2001)
A spermine-coupled cholesterol metabolite from the shark with potent appetite suppressant and antidiabetic properties. Int. J. Obes. 25, 689697.

(32) Zasloff, M., Adams, A. P., Beckerman, B., Campbell, A., Han, Z., Luijten, E., Meza, I., Julander, J., Mishra, A., Qu, W., Taylor, J. M., Weaver, S. C., and Wong, G. C. L. (2011) Squalamine as a broadspectrum systemic antiviral agent with therapeutic potential. Proc. Natl. Acad. Sci. U. S. A. 108, 15978-15983.

(33) Rao, M. N., Shinnar, A. E., Noecker, L. A., Chao, T. L., Feibush, B., Snyder, B., Sharkansky, I., Sarkahian, A., Zhang, X., Jones, S. R., Kinney, W. A., and Zasloff, M. (2000) Aminosterols from the Dogfish Shark Squalus acanthias. J. Nat. Prod. 63, 631-635.

(34) Sills, A. K., Williams, J. I., Tyler, B. M., Epstein, D. S., Sipos, E. P., Davis, J. D., McLane, M. P., Pitchford, S., Cheshire, K., Gannon, F. H., Kinney, W. A., Chao, T. L., Donowitz, M., Laterra, J., Zasloff, M., and Brem, H. (1998) Squalamine inhibits angiogenesis and solid tumor growth in vivo and perturbs embryonic vasculature. Cancer Res. 58, 2784-2792.

(35) Krishnan, N., Koveal, D., Miller, D. H., Xue, B., Akshinthala, S. D., Kragelj, J., Jensen, M. R., Gauss, C.-M., Page, R., Blackledge, M., Muthuswamy, S. K., Peti, W., and Tonks, N. K. (2014) Targeting the disordered C terminus of PTP1B with an allosteric inhibitor. Nat. Chem. Biol. 10, 558-566.

(36) Qin, Z, Zhou, X, Pandey, N.R., Vecchiarelli, H.A., Stewart, C.A., Zhang, X., Lagace, D.C., Brunel, J.M., Béique, J.C., Stewart, A.F., Hill, M.N., and Chen, H.H. Chronic stress induces anxiety via an amygdalar intracellular cascade that impairs endocannabinoid signaling. Neuron 85(6):1319-31.

(37) Lantz, K. A., Hart, S. G. E., Planey, S. L., Roitman, M. F., RuizWhite, I. A., Wolfe, H. R., and McLane, M. P. (2010) Inhibition of PTP1B by trodusquemine (MSI-1436) causes fat-specific weight loss in diet-induced obese mice. Obesity 18, 1516-1523.

(38) Ahima, R. S., Patel, H. R., Takahashi, N., Qi, Y., Hileman, S. M., and Zasloff, M. A. (2002) Appetite suppression and weight reduction by a centrally active aminosterol. Diabetes 51, 2099-2104.

(39) Smith, A. M., Maguire-Nguyen, K. K., Rando, T. A., Zasloff, M. A., Strange, K. B., and Yin, V. P. (2017) The protein tyrosine phosphatase $1 \mathrm{~B}$ inhibitor MSI-1436 stimulates regeneration of heart and multiple other tissues. NPJ. Regen Med. 2, 57.

(40) Yeung, T., Gilbert, G. E., Shi, J., Silvius, J., Kapus, A., and Grinstein, S. (2008) Membrane phosphatidylserine regulates surface charge and protein localization. Science 319, 210-212.

(41) Brown, J. W. P., Buell, A. K., Michaels, T. C. T., Meisl, G., Carozza, J., Flagmeier, P., Vendruscolo, M., Knowles, T. P. J., Dobson, C. M., and Galvagnion, C. (2016) $\beta$-Synuclein suppresses both the initiation and amplification steps of $\alpha$-synuclein aggregation via competitive binding to surfaces. Sci. Rep. 6, 36010.

(42) Gaspar, R., Meisl, G., Buell, A. K., Young, L., Kaminski, C. F., Knowles, T. P. J., Sparr, E., and Linse, S. (2017) Acceleration of $\alpha$ synuclein aggregation. Amyloid 24, 20-21.

(43) Fusco, G., Pape, T., Stephens, A. D., Mahou, P., Costa, A. R. Kaminski, C. F., Kaminski Schierle, G. S., Vendruscolo, M., Veglia, G., Dobson, C. M., and De Simone, A. (2016) Structural basis of synaptic vesicle assembly promoted by $\alpha$-synuclein. Nat. Commun. 7, 12563.

(44) Cohen, S. I. A., Arosio, P., Presto, J., Kurudenkandy, F. R, Biverstal, H., Dolfe, L., Dunning, C., Yang, X., Frohm, B., Vendruscolo, M., Johansson, J., Dobson, C. M., Fisahn, A., Knowles, T. P. J., and Linse, S. (2015) The molecular chaperone Brichos breaks the catalytic cycle that generates toxic $\mathrm{A} \beta$ oligomers. Nat. Struct. Mol. Biol. 22, 207-213.

(45) Seidler, J., McGovern, S. L., Doman, T. N., and Shoichet, B. K. (2003) Identification and prediction of promiscuous aggregating inhibitors among known drugs. J. Med. Chem. 46, 4477-4486.

(46) Cremades, N., Cohen, S. I. A., Deas, E., Abramov, A. Y., Chen, A. Y., Orte, A., Sandal, M., Clarke, R. W., Dunne, P., Aprile, F. A., Bertoncini, C. W., Wood, N. W., Knowles, T. P. J., Dobson, C. M., and Klenerman, D. (2012) Direct observation of the interconversion of normal and toxic forms of $\alpha$-synuclein. Cell 149, 1048-1059.

(47) Chen, S. W., Drakulic, S., Deas, E., Ouberai, M., Aprile, F. A., Arranz, R., Ness, S., Roodveldt, C., Guilliams, T., De-Genst, E. J., 
Klenerman, D., Wood, N. W., Knowles, T. P. J., Alfonso, C., Rivas, G., Abramov, A. Y., Valpuesta, J. M., Dobson, C. M., and Cremades, N. (2015) Structural characterization of toxic oligomers that are kinetically trapped during $\alpha$-synuclein fibril formation. Proc. Natl. Acad. Sci. U. S. A. 112, E1994-2003.

(48) Flagmeier, P., De, S., Wirthensohn, D. C., Lee, S. F., Vincke, C., Muyldermans, S., Knowles, T. P. J., Gandhi, S., Dobson, C. M., and Klenerman, D. (2017) Ultrasensitive measurement of Ca (2+) influx into lipid vesicles induced by protein aggregates. Angew. Chem., Int. Ed. 56, 7750-7754.

(49) Evangelisti, E., Cascella, R., Becatti, M., Marrazza, G., Dobson, C. M., Chiti, F., Stefani, M., and Cecchi, C. (2016) Binding affinity of amyloid oligomers to cellular membranes is a generic indicator of cellular dysfunction in protein misfolding diseases. Sci. Rep. 6, 32721.

(50) Eisenberg, T., Knauer, H., Schauer, A., Büttner, S., Ruckenstuhl, C., Carmona-Gutierrez, D., Ring, J., Schroeder, S., Magnes, C., Antonacci, L., Fussi, H., Deszcz, L., Hartl, R., Schraml, E., Criollo, A., Megalou, E., Weiskopf, D., Laun, P., Heeren, G., Breitenbach, M., Grubeck-Loebenstein, B., Herker, E., Fahrenkrog, B., Fröhlich, K.-U., Sinner, F., Tavernarakis, N., Minois, N., Kroemer, G., and Madeo, F. (2009) Induction of autophagy by spermidine promotes longevity. Nat. Cell Biol. 11, 1305-1314.

(51) Bauer, J. H., Morris, S. N. S., Chang, C., Flatt, T., Wood, J. G., and Helfand, S. L. (2008) dSir2 and Dmp53 interact to mediate aspects of CR-dependent life span extension in D. melanogaster. Aging 1, 38-48. (52) Hamilton, B., Dong, Y., Shindo, M., Liu, W., Odell, I., Ruvkun, G., and Lee, S. S. (2005) A systematic RNAi screen for longevity genes in C. elegans. Genes Dev. 19, 1544-1555.

(53) Sarin, S., Prabhu, S., O'Meara, M. M., Pe'er, I., and Hobert, O. (2008) Caenorhabditis elegans mutant allele identification by wholegenome sequencing. Nat. Methods 5, 865-867.

(54) Kim, Y., and Sun, H. (2007) Functional genomic approach to identify novel genes involved in the regulation of oxidative stress resistance and animal lifespan. Aging Cell 6, 489-503.

(55) Dillin, A., Hsu, A.-L., Arantes-Oliveira, N., Lehrer-Graiwer, J., Hsin, H., Fraser, A. G., Kamath, R. S., Ahringer, J., and Kenyon, C. (2002) Rates of behavior and aging specified by mitochondrial function during development. Science 298, 2398-2401.

(56) Lee, S. S., Kennedy, S., Tolonen, A. C., and Ruvkun, G. (2003)

DAF-16 target genes that control C. elegans life-span and metabolism. Science 300, 644-647.

(57) Jorgensen, E. M., and Mango, S. E. (2002) The art and design of genetic screens: caenorhabditis elegans. Nat. Rev. Genet. 3, 356-369.

(58) Ayyadevara, S., Balasubramaniam, M., Johnson, J., Alla, R., Mackintosh, S. G., and Reis, R. J. S. (2016) PIP(3)-binding proteins promote age-dependent protein aggregation and limit survival in $\mathrm{C}$. elegans. Oncotarget 7, 48870-48886.

(59) Johnson, C. S. (1999) Diffusion ordered nuclear magnetic resonance spectroscopy: Principles and applications. Prog. Nucl. Magn. Reson. Spectrosc. 34, 203-256.

(60) Wu, D. H., Chen, A. D., and Johnson, C. S. (1995) An improved diffusion-ordered spectroscopy experiment incorporating bipolargradient pulses. J. Magn. Reson., Ser. A 115, 260-264.

(61) Stejskal, E. O., and Tanner, J. E. (1965) Spin diffusion measurements: spin echoes in the presence of a time-dependent field gradient. J. Chem. Phys. 42, 288-292.

(62) Capitini, C., Conti, S., Perni, M., Guidi, F., Cascella, R., De Poli, A., Penco, A., Relini, A., Cecchi, C., and Chiti, F. (2014) TDP-43 inclusion bodies formed in bacteria are structurally amorphous, non-amyloid and inherently toxic to neuroblastoma cells. PLoS One 9, e86720.

(63) Di Natale, C., Scognamiglio, P. L., Cascella, R., Cecchi, C., Russo, A., Leone, M., Penco, A., Relini, A., Federici, L., Di Matteo, A., Chiti, F., Vitagliano, L., and Marasco, D. (2015) Nucleophosmin contains amyloidogenic regions that are able to form toxic aggregates under physiological conditions. FASEB J. 29, 3689-3701.

(64) Brenner, S. (1974) The genetics of Caenorhabditis elegans. Genetics 77, 71-94.

(65) Van Ham, T. J., Thijssen, K. L., Breitling, R., Hofstra, R. M. W., Plasterk, R. H. A., and Nollen, E. A. A. (2008) C. elegans model identifies genetic modifiers of $\alpha$-synuclein inclusion formation during aging. PLoS Genet. 4, e1000027-11.

(66) Perni, M., Challa, P. K., Kirkegaard, J. B., Limbocker, R., Koopman, M., Hardenberg, M. C., Sormanni, P., Müller, T., Saar, K. L., Roode, L. W. Y., Habchi, J., Vecchi, G., Fernando, N. W., Casford, S., Nollen, E. A. A., Vendruscolo, M., Dobson, C. M., and Knowles, T. P. J. (2018) Massively parallel C. elegans tracking provides multi-dimensional fingerprints for phenotypic discovery. J. Neurosci. Methods, DOI: 10.1016/j.jneumeth.2018.02.005.

(67) Van der Goot, A. T., Zhu, W., Vazquez-Manrique, R. P., Seinstra, R. I., Dettmer, K., Michels, H., Farina, F., Krijnen, J., Melki, R., Buijsman, R. C., Ruiz Silva, M., Thijssen, K. L., Kema, I. P., Neri, C., Oefner, P. J., and Nollen, E. A. A. (2012) Delaying aging and the aging-associated decline in protein homeostasis by inhibition of tryptophan degradation. Proc. Natl. Acad. Sci. U. S. A. 109, 14912-14917. 